\title{
Relative Index Theorems and Supersymmetric Scattering Theory
}

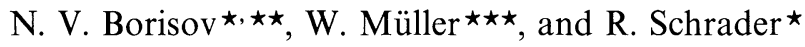 \\ Institut für Theoretische Physik, Freie Universität Berlin, Arnimallee 14, D-1000 Berlin 33, \\ Federal Republic of Germany
}

\begin{abstract}
We discuss supersymmetric scattering theory and employ Krein's theory of spectral shift functions to investigate supersymmetric scattering systems. This is the basis for the derivation of relative index theorems on some classes of open manifolds. As an example we discuss the de Rham complex for obstacles in $\mathbb{R}^{N}$ and asymptotically flat manifolds. It is shown that the absolute or relative Euler characteristic of an obstacle in $\mathbb{R}^{N}$ may be obtained from scattering data for the Laplace operator on forms with absolute or relative boundary conditions respectively. In the case of asymptotically flat manifolds we obtain the Chern-Gauss-Bonnet theorem for the $L^{2}$-Euler characteristic.
\end{abstract}

\section{Introduction}

Supersymmetry is a recent concept of quantum field theory which has also interesting applications in mathematics. Recall that a supersymmetry theory consists of a Hilbert space $\mathscr{H}$ together with a unitary involution $\tau$ in $\mathscr{H}$ [in physics $\tau$ is usually denoted by $\left.(-1)^{F}\right]$ and selfadjoint operators $Q_{i}, i=1, \ldots, N$, acting in $\mathscr{H}$ and satisfying

$$
\begin{gathered}
\tau Q_{i}+Q_{i} \tau=0, \quad i=1, \ldots, N \\
Q_{i} Q_{j}+Q_{j} Q_{i}=0, \quad i \neq j .
\end{gathered}
$$

Moreover, the Hamiltonian $H$ of the theory satisfies

$$
H=Q_{i}^{2}
$$

for each $i . Q_{i}$ is called supercharge and $H$ a Hamiltonian with supersymmetry.

^ Institut für Theorie der Elementarteilchen, Freie Universität Berlin, D-1000 Berlin, FRG

$\star \star$ On leave of absence from Institute of Physics, Leningrad State University, Leningrad

$\star \star \star$ Karl-Weierstrass-Institut für Mathematik, Akademie der Wissenschaften der DDR, Berlin, German Democratic Republic 
In this paper we shall investigate supersymmetric scattering systems consisting of two supersymmetric Hamiltonians $H=Q^{2}, H_{0}=Q_{0}^{2}$ acting in a given Hilbert space $\mathscr{H}$ (the involution $\tau$ is assumed to be the same for $Q$ and $Q_{0}$ ) so that the Møller operators $W_{ \pm}\left(H, H_{0}\right)$ exist, are complete and intertwine the supercharges $Q$ and $Q_{0}$. This implies that the supercharge $Q_{0}$ commutes with the associated scattering operator $S$. This fact is of particular interest for the applications we have in mind.

Supersymmetric scattering systems arise both in mathematics and physics. For example, in mathematics supersymmetric scattering theory is closely related to index problems for elliptic operators on noncompact manifolds. Recall that an elliptic differential operator $D: C^{\infty}\left(X, E^{+}\right) \rightarrow C^{\infty}\left(X, E^{-}\right)$on a closed Riemannian manifold $X$ gives rise to a supersymmetric Hamiltonian $H=\left(\begin{array}{cc}D^{*} D & 0 \\ 0 & D D^{*}\end{array}\right)$ acting in the Hilbert space $L^{2}\left(X, E^{+}\right) \oplus L^{2}\left(X, E^{-}\right)$with involution $\tau=\left(\begin{array}{cc}I & 0 \\ 0 & -I\end{array}\right)$. Moreover, the index of $D$ is given by

$$
\text { Ind } D=\operatorname{Tr}\left(\tau e^{-t H}\right)
$$

(cf. [1]). In fact, due to supersymmetry the positive spectrum cancels out on the right-hand side. If we are dealing with elliptic differential operators on noncompact manifolds then, in general, the corresponding Hamiltonian $H$ will have a nonempty continuous spectrum. In particular, $\exp (-t H)$ is not of trace class. However, many index problems on noncompact manifolds give rise to pairs $\left(H, H_{0}\right)$ of supersymmetric Hamiltonians where $H_{0}$ can be considered as the free Hamiltonian describing the continuous spectrum of $H$. In these cases $\exp (-t H)$ $-\exp \left(-t H_{0}\right)$ is of trace class and in place of (1.1) we have to investigate the following supertrace:

$$
\operatorname{Tr}\left(\tau\left(e^{-t H}-e^{-t H_{0}}\right)\right)
$$

Again, due to supersymmetry one may expect that the positive spectrum cancels out in (1.2). In this case we get a relative index theorem. The essential problem is to investigate the contribution of the continuous spectrum to (1.2). This is where supersymmetric scattering theory comes into play. Basic is the commutation relation mentioned above for the scattering operator $S$ and the free supercharge $Q_{0}$. In a hidden form this occurs already in the work of Barbasch and Moscovici [8] on the $L^{2}$-index of elliptic operators on locally symmetric spaces of real rank one. They use a sort of commutation relations for the constant terms of the Eisenstein series to treat the contribution of the continuous spectrum to the $L^{2}$-index. The constant terms of the Eisenstein series are closely related to the scattering matrix with respect to some particular free Hamiltonian. The relations they are using are not exactly commutation relations. This is due to the fact that their free Hamiltonian is not supersymmetric (cf. [41]).

In physics supersymmetric scattering theory is closely connected with supersymmetric quantum mechanics. For a special case supersymmetric scattering theory was originally discussed in [3] (for alternative discussions of scattering theory in supersymmetric quantum mechanics, see e.g. $[11,13,23,29]$ and the 
references quoted there). In particular, there is a close relationship to the phenomenon of fractionally charged states and the fractional Witten index $[2,11$, 13]. In [52] Witten introduced a quantity which counts the difference of the number of bosonic and fermionic zero-energy modes of the Hamiltonian of the theory. In the case the Hamiltonian has a non-empty continuous spectrum a regularized Witten index has been introduced by several authors (cf. [13]). In this context many supersymmetric quantum mechanical models have been discussed in the literature $[2,11,13]$. A model which has been reconsidered by many authors is

$$
\begin{gathered}
D=d / d x+\Phi, \quad \lim _{x \rightarrow \pm \infty} \Phi(x)=\Phi_{ \pm}, \\
H=\left(\begin{array}{cc}
H_{1} & 0 \\
0 & H_{2}
\end{array}\right), \quad H_{j}=-d^{2} / d x^{2}+\Phi^{2}+(-1)^{j} \Phi^{\prime}, \quad j=0,1 .
\end{gathered}
$$

The discussion of all these models fits completely in our framework and our approach puts the regularized Witten index in the context of relative index theorems.

In the first section we study abstract supersymmetric scattering systems. The main implication of supersymmetry is that the superdeterminant of the on-shell $S$-matrix is equal to 1 . Moreover, if the resulting phase shift functions (which are functions of the energy) in the bosonic and fermionic sectors exist and are continuous, then modulo the factor $\pi$ their difference is shown to be an integer, called the supersymmetric scattering index. We continue by considering pairs of supersymmetric Hamiltonians $H=Q^{2}, H_{0}=Q_{0}^{2}$, so that

$$
e^{-t H}-e^{-t H_{0}} \text { and } Q e^{-t H}-Q_{0} e^{-t H_{0}}
$$

are of the trace class for each $t>0$.

This assumption implies that $\left(H, H_{0}\right)$ is a supersymmetric scattering system. Condition (1.4) enables us to employ Krein's theory of spectral shift functions (see e.g. [9]). If $\xi\left(E ; H, H_{0}\right)$ denotes the spectral shift function and $S\left(E ; H, H_{0}\right)$ the onshell scattering matrix associated to $H$ and $H_{0}$, then the basic fact we shall employ is the following equation:

$$
\operatorname{det} S\left(E ; H, H_{0}\right)=e^{2 \pi i \xi\left(E ; H, H_{0}\right)}, \quad E \in \sigma_{\mathrm{ac}}(H) .
$$

This fact, dating back to the observation of Beth and Uhlenbeck [10] that the second virial coefficient is related to the phase shift function, has been considered by Birman, Buslaev, Faddejev, Krein and is the basis of several trace formulas. Let $\xi_{+}\left(E ; H, H_{0}\right)$ and $\xi_{-}\left(E ; H, H_{0}\right)$ be the bosonic and fermionic part of the spectral shift function respectively. Under the assumption that $\xi_{+}\left(E ; H, H_{0}\right)-\xi_{-}\left(E ; H, H_{0}\right)$ is continuous in $E>0$ and the zero eigenvalue of $H$ and $H_{0}$ is of finite multiplicity, we obtain our main result

$$
\operatorname{Trace}\left(\tau\left(e^{-t H}-e^{-t H_{0}}\right)\right)=n^{\text {point }}(H)-n^{\text {point }}\left(H_{0}\right)+n^{c}\left(H, H_{0}\right) .
$$

Here $n^{\text {point }}(H)=n_{+}(H)-n_{-}(H)$, where $n_{+}(H)$ [respectively $n_{-}(H)$ ] counts the number of bosonic (respectively fermionic) zero-energy states. $n^{\text {point }}\left(H_{0}\right)$ is defined similarly and $n^{c}\left(H, H_{0}\right)$ is an integer determined by the continuous spectrum. If $H$ and $H_{0}$ are Hamiltonians associated to differential operators $D$ and $D_{0}$, then we 
can replace the left-hand side by integrals involving the pointwise trace of the corresponding heat kernels. Moreover, in this case one has $n^{\text {point }}(H)=L^{2}-\operatorname{Ind} D$ and $n^{\text {point }}\left(H_{0}\right)=L^{2}-\operatorname{Ind} D_{0}$. Here

$$
L^{2}-\operatorname{Ind} D=\operatorname{dim}\left(\operatorname{ker} D \cap L^{2}\right)-\operatorname{dim}\left(\operatorname{ker} D^{*} \cap L^{2}\right),
$$

and similar for $D_{0}$. Based on these observations one can derive index theorems for elliptic operators on noncompact manifolds by investigating the asymptotic behaviour as $t \rightarrow 0$ of the left-hand side of (1.5). For example, this approach can be used to prove an $L^{2}$-index theorem for generalized Dirac operators on $\mathbb{Q}$-rank one locally symmetric spaces [41] (cf. also [8, 12, 18, 31, 41, 48, 49, 54] for related results concerning index problems on noncompact manifolds). In general, it is difficult to determine the number $n^{c}\left(H, H_{0}\right)$ occurring in (1.5). It is related to zeroenergy resonances.

If we apply (1.5) to the models arising in supersymmetric quantum mechanics, we get index formulas which can be used to discuss the regularized Witten index. We elaborate on this by discussing (1.4) explicitly.

In the rest of the paper we discuss several examples of supersymmetric scattering systems. In Sect. 3 we consider scattering by an obstacle in $\mathbb{R}^{N}$ (see e.g. $[16,17,33,40])$. This means that the Hamiltonian $H$ is the Laplacian acting on differential forms in the complement of the obstacle with absolute or relative boundary conditions. The supersymmetric scattering index turns out to be minus the absolute or relative Euler characteristic of the obstacle respectively. Thus the Euler characteristic of the obstacle can be extracted out of the scattering data. We observe that scattering on 1-forms is related to scattering of electromagnetic waves by obstacles (see e.g. $[39,51]$ ).

In Sect. 4 we consider manifolds which are euclidean at infinity. One may regard this example as the scattering of plane waves by changing the topology of $\mathbb{R}^{N}$. Again we discuss the Laplace operator acting on differential forms. In this case the supersymmetric scattering index turns out to be the $L^{2}$-index of the GaussBonnet operator which is the alternating sum of the dimensions of the spaces of square integrable harmonic $p$-forms. This is proved by converting the $L^{2}$-problem into a non-local boundary value problem similar to [7]. The same result could be obtained by analysing the threshold behaviour of the spectral shift function.

By a simple stability argument we extend this result to asymptotically euclidean manifolds. This gives a new proof of the Chern-Gauss-Bonnet theorem for these manifolds which is due to Stern [48]. Our result is slightly more general, including in particular the two-dimensional case.

Throughout this paper we employ the following notation. $\Omega(M)$ denotes the space of smooth complex forms on a smooth manifold $M$. The subspace of smooth $p$-forms is denoted by $\Omega^{p}(M)$, whereas the space of even and odd smooth forms is denoted by $\Omega_{+}(M)$ and $\Omega_{-}(M)$, respectively. Finally $\Omega_{c}(M)$ is the subspace of smooth forms with compact support.

\section{Abstract Supersymmetric Scattering Theory}

We will consider the following general set-up. Assume there is a Hilbert space $\mathscr{H}$ with a selfadjoint (s.a.) and hence unitary involution $\tau$. The eigenspaces $\mathscr{H}_{ \pm}$for the 
eigenvalues $\tau=+1$ and $\tau=-1$ are called the bosonic and fermionic subspaces respectively. Trivially we have $\mathscr{H}=\mathscr{H}_{+} \oplus \mathscr{H}_{-}$.

Definition 2.1. A selfadjoint operator $Q$ in $\mathscr{H}$ is called a supercharge with respect to $\tau$ if $\tau$ leaves the domain $\mathscr{D}(Q)$ of $Q$ invariant and if $\tau$ anticommutes with $Q$ on $\mathscr{D}(Q)$. The selfadjoint operator $H=Q^{2} \geqq 0$ is called the associated Hamiltonian. Any operator $H$ of this form for some $Q$ and $\tau$ is called a Hamiltonian with supersymmetry.

By the anticommuting properties of $Q$ and $\tau$, if $\varphi \in \mathscr{D}(Q)$ is bosonic, then $Q \varphi$ is fermionic and vice versa. This is the motivation for calling $Q$ a supercharge.

The following example will be our starting point, when we construct examples for a supersymmetric scattering theory.

Example 2.2. On a compact, oriented closed Riemannian manifold $M$, let $Q=d+\delta$, where $d$ is the exterior derivative on forms and $\delta$ is the coderivative. $\mathscr{H}$ is the space of square integrable forms. Then $H=Q^{2}$ is the Laplace operator on $\mathscr{H}$. Let $P$ be the linear operator which is $p$ on $p$-forms. As a first choice for $\tau$ we take $(-1)^{P} . \mathscr{H}_{+}$is the subspace of even forms and $\mathscr{H}_{-}$is the subspace of odd forms. This form of $\tau$ is relevant in the context of the Rham complexes. As a second example for $\tau$ we will take $\tau=* \circ i^{P(P-1)+N / 2}$ in case the manifold has even dimension $N$. Here $*$ is the Hodge duality operator. This choice will lead us to the signature operator (see the discussion after Example 2.3).

Example 2.3. Let $L$ be a closed linear map with dense domain from one Hilbert space $\mathscr{H}_{+}$to another Hilbert space $\mathscr{H}_{-}$. Let $L^{*}: \mathscr{H}_{-} \rightarrow \mathscr{H}_{+}$be the adjoint. Since $L$ is closed, $L^{*}$ is densely defined $([37]$, p. 168) and closed. We form the space $\mathscr{H}=\mathscr{H}_{+} \oplus \mathscr{H}_{-}$. Let $\tau$ be the linear operator in $\mathscr{H}$ which is +1 on $\mathscr{H}_{+}$and -1 on $\mathscr{H}_{-}$. Now let the selfadjoint operator $Q$ in $\mathscr{H}$ be given in matrix form as $\left(\begin{array}{cc}0 & L^{*} \\ L & 0\end{array}\right)$. Then $H=Q^{2}$ takes the form $\left(\begin{array}{cc}L^{*} L & 0 \\ 0 & L L^{*}\end{array}\right)$.

The following decomposition of $Q$ shows that Example 2.3 covers the general situation. If $Q$ is a supercharge with respect to $\tau$, then $\mathscr{D}(Q)$ $=\left(\mathscr{D}(Q) \cap \mathscr{H}_{+}\right) \oplus\left(\mathscr{D}(Q) \cap \mathscr{H}_{-}\right)$, and there is the unique decomposition

$$
Q=P_{-} Q P_{+}+P_{+} Q P_{-}=Q_{+}+Q_{-},
$$

$P_{ \pm}=1 / 2(1 \pm \tau)$ being the orthogonal projections onto $\mathscr{H}_{+}$and $\mathscr{H}_{-}$respectively. We have $Q_{+}^{2}=Q_{-}^{2}=0, Q_{+}^{*}=Q_{-}$, and $H=Q_{+} Q_{-}+Q_{-} Q_{+}$. Thus in Example 2.1 with $\tau=(-1)^{P}, Q_{+}$is $(d+\delta)$ on even forms and zero otherwise, while $Q_{-}$is $(d+\delta)$ on odd forms and zero otherwise. If $\tau$ is of the second form in Example 2.1, then $Q_{+}$ is the signature operator. In Example 2.3 we have $Q_{+}=\left(\begin{array}{ll}0 & 0 \\ L & 0\end{array}\right)$ and $Q_{-}=\left(\begin{array}{ll}0 & L^{*} \\ 0 & 0\end{array}\right)$

Note that the s.a. operator

$$
Q^{\prime}=i\left(P_{-} Q P_{+}-P_{+} Q P_{-}\right)=i Q \tau=-i \tau Q
$$


is also a supercharge with respect to $\tau$ such that

$$
Q^{2}=Q^{\prime 2}, \quad Q Q^{\prime}+Q^{\prime} Q=0 \quad \text { on } \mathscr{D}(H) .
$$

Thus supercharges always occur in pairs. Conversely given two s.a. operators $Q$ and $Q^{\prime}$ satisfying (2.2), then by a little algebra it is easy to construct complementary subspaces $\mathscr{H}_{+}$and $\mathscr{H}_{-}$with a resulting unitary involution $\tau$ such that $Q$ and $Q^{\prime}$ are supercharges with respect to this $\tau$ and are related by (2.1).

The following consideration was one of our motivations to look at supersymmetric scattering theory. Assume there is a supercharge $Q$ with respect to $\tau$. Let $\mathscr{H}(0)$ be the closed subspace of $\mathscr{H}$ consisting of all $\varphi \in \mathscr{D}\left(H=Q^{2}\right)$ with $H \varphi=0$, i.e. $\mathscr{H}(0)$ is the eigenspace for the eigenvalue 0 , whenever 0 is an eigenvalue. Obviously

$$
\mathscr{H}(0)=\left(\mathscr{H}(0) \cap \mathscr{H}_{+}\right) \oplus\left(\mathscr{H}(0) \cap \mathscr{H}_{-}\right)=\mathscr{H}_{+}(0) \oplus \mathscr{H}_{-}(0) .
$$

We recall the following lemma for the so-called Witten index [52].

Lemma 2.4. Assume exp- $t H$ is of trace class for all $t>0$ ( such that $\mathscr{H}(0)$ has finite dimension). Then for all $t>0$ the supersymmetric trace of the semigroup satisfies the relation

$$
\text { Trace } \tau(\exp -t H)=\operatorname{dim} \mathscr{H}_{+}(0)-\operatorname{dim} \mathscr{H}_{-}(0) \text {. }
$$

Proof. The proof is well known and a natural extension of arguments familiar from heat equation methods. Let $0 \neq \varphi \in \mathscr{H}_{+}$be an eigenvector of $H$ with eigenvalue $\varepsilon>0$. Then $Q \varphi$ is also an eigenvector of $H$ with eigenvalue $\varepsilon>0$. But now $Q \varphi \in \mathscr{H}_{-}$. Also since $Q(Q \varphi)=Q^{2} \varphi=H \varphi=\varepsilon \varphi$, we have that $Q \varphi \neq 0$ and that the linear correspondence $\varphi \rightarrow Q \varphi$ is one-to-one on the set of eigenvector with eigenvalue $\varepsilon>0$. Hence on the left-hand side of (2.3) all contributions from nonvanishing eigenvalues cancel and Lemma 2.4 is proved.

Now Lemma 2.4 says in particular that under the given assumptions the supersymmetric trace is an integer. Applying this to Example 2.3 gives the starting point for the heat kernel proof [6] of the Atiyah-Singer index theorem (see e.g. [30]). This index theorem is applicable to operators on compact manifolds only. In the situation of noncompact manifold the corresponding heat kernels $\exp -t Q^{2}$ will in general not be of trace class.

However, we will present cases where $Q$ is a perturbation of another supercharge $Q_{0}$, which we will call a free supercharge with free Hamiltonian $H_{0}$, and such that exp- $t H-\exp -t H_{0}$ is of trace class. The natural question is then what the value of the corresponding supertrace is. It turns out that scattering theory often can predict this value to be an integer independent of $t$. We now outline the arguments leading to such a result and start with a definition of a supersymmetric scattering theory.

Definition 2.5. A supersymmetric scattering theory in a Hilbert space $\mathscr{H}$ with a unitary involution $\tau$ is given by a pair $\left(Q, Q_{0}\right)$ with the following properties:

(a) $Q$ and $Q_{0}$ are supercharges with respect to $\tau$.

(b) For the associated Hamiltonians $H=Q^{2}$ and $H_{0}=Q_{0}^{2}$ the Møller operators $W^{ \pm}\left(H, H_{0}\right)=s-\lim _{t \rightarrow \pm \infty} e^{i t H} e^{-i t H_{0}} P_{\text {ac }}\left(H_{0}\right)$ exist, and are complete. 
(c) $W^{ \pm}\left(H, H_{0}\right)$ are intertwining operators for $Q$ and $Q_{0}$ :

$$
Q W^{ \pm}\left(H, H_{0}\right)=W^{ \pm}\left(H, H_{0}\right) Q_{0} \quad \text { on } \quad \mathscr{D}\left(Q_{0}\right) \cap \mathscr{H}_{0, \mathrm{ac}} .
$$

$P_{\text {ac }}\left(H_{0}\right)$ is the orthogonal projection onto the absolutely continuous subspace $\mathscr{H}_{0, \text { ac }}$ of $H_{0}$. Completeness means that the relation $\operatorname{Ran} W^{+}\left(H, H_{0}\right)$ $=\operatorname{Ran} W^{-}\left(H, H_{0}\right)$ holds such that both $W^{+}\left(H, H_{0}\right)$ and $W^{-}\left(H, H_{0}\right)$ are unitary operators from $\mathscr{H}_{0, \text { ac }}$ to $\mathscr{H}_{\mathrm{ac}}=P_{\mathrm{ac}}(H) \mathscr{H}$, the absolutely continuous subspace of $H$.

Condition (c) is the new supersymmetric ingredient for scattering theory, which was first introduced in [3]. This definition has an immediate extension to the two Hilbert space scattering theory [36]. Namely consider the situation where $Q$ is a supercharge with respect to $\tau$ in $\mathscr{H}$ and $Q_{0}$ a supercharge with respect to $\tau_{0}$ in $\mathscr{H}_{0}$. Let $J: \mathscr{H}_{0} \rightarrow \mathscr{H}$ be an intertwining operator for $\tau$ and $\tau_{0}$, i.e. $J \tau_{0}=\tau J$. For a supersymmetric scattering theory we now require the Møller operators

$$
W^{ \pm}\left(H, H_{0} ; J\right)=\underset{t \rightarrow \pm \infty}{s-\lim } e^{i t H} J e^{-i t H_{0}} P_{\text {ac }}\left(H_{0}\right)
$$

to exist and to be complete. By construction these operators are intertwining operators for $H$ and $H_{0}$ as well as for $\tau$ and $\tau_{0}$. In addition we require the Møller operators to be intertwining operators for $Q$ and $Q_{0}$ on $\mathscr{D}\left(Q_{0}\right) \cap \mathscr{H}_{0 \text {,ac. }}$

Note that the conditions of Definition 2.5 are met, if the Møller operators $W^{ \pm}\left(Q, Q_{0}\right)$ for $Q$ and $Q_{0}$ exist and are complete. Hence the following lemma, which will be applied in the theory of obstacles and in Sect. 4, will provide us with a sufficient condition for the existence of a supersymmetric scattering theory.

Lemma 2.6. Assume $Q$ and $Q_{0}$ are supercharges in $\mathscr{H}$ with respect to $\tau$ such that $Q \exp -t Q^{2}-Q_{0} \exp -t Q_{0}^{2}$ is of trace class for all $t>0$. Then $Q$ and $Q_{0}$ define $a$ supersymmetric scattering theory.

Proof. By the Kato-Rosenblum theory (see e.g. [37]) for any $t>0$ the Møller operators $W^{ \pm}\left(Q\right.$ exp- $t Q^{2}, Q_{0}$ exp- $\left.t Q_{0}^{2}\right)$ exist and are complete. By the Birman-Kato invariance principle of the wave operators (see [35]) $W^{ \pm}\left(Q, Q_{0}\right)$ exist and are complete. By the same invariance principle, $W^{ \pm}\left(H, H_{0}\right)$ exist such that if $P\left(Q_{0} \geqq 0\right)$ is the orthogonal projection onto the absolute continuous subspace on which $Q_{0} \geqq 0$, then $W^{+}\left(H, H_{0}\right) u=W^{+}\left(Q, Q_{0}\right) u$ whenever $P\left(Q_{0} \geqq 0\right) u=u$ and $W^{+}\left(H, H_{0}\right) u$ $=W^{-}\left(Q, Q_{0}\right) u$ whenever $P\left(Q_{0} \geqq 0\right) u=0$. From this the required intertwining properties c) of $W^{ \pm}\left(H, H_{0}\right)$ for $Q$ and $Q_{0}$ follow from the intertwining properties of $W^{ \pm}\left(Q, Q_{0}\right)$ for $Q$ and $Q_{0}$.

We turn to a discussion of the resulting $S$-Matrix, defined as

$$
S\left(H, H_{0}\right)=W^{+}\left(H, H_{0}\right) * W^{-}\left(H, H_{0}\right),
$$

which by the above completeness assumption is a unitary operator on $\mathscr{H}_{0, \mathrm{ac}}$ with the properties

$$
\begin{gathered}
\tau S\left(H, H_{0}\right)=S\left(H, H_{0}\right) \tau \\
Q_{0} S\left(H, H_{0}\right)=S\left(H, H_{0}\right) Q_{0} \quad \text { on } \quad \mathscr{D}\left(Q_{0}\right) \cap \mathscr{H}_{0, \mathrm{ac}} .
\end{gathered}
$$

By (2.5) we may write

$$
S\left(H, H_{0}\right)=S_{+}\left(H, H_{0}\right) \oplus S_{-}\left(H, H_{0}\right)
$$


with respect to the decomposition $\mathscr{H}_{0, \text { ac }}=\mathscr{H}_{0, \mathrm{ac}+} \oplus \mathscr{H}_{0 \text {, ac }-}$ into bosonic and fermionic parts. $Q_{0}$ is of course again a map from $\mathscr{H}_{0, \text { ac }}$ into $\mathscr{H}_{0, \text { ac }-}$ and vice versa.

We now elaborate on these relations. Since $S\left(H, H_{0}\right)$ commutes with $H_{0}$ and $Q_{0}$, the spectral decomposition of $H_{0 \text {, ac }}$ (the absolutely continuous part of $H_{0}$ ) on $\mathscr{H}_{0 \text {, ac }}$,

$$
H_{0, \mathrm{ac}}=\int E d P_{\mathrm{ac}}(E) \text {, }
$$

defines a corresponding spectral decomposition of $S$ and $Q_{0}$ in the form

$$
S\left(H, H_{0}\right)=\int S\left(E ; H, H_{0}\right) d P_{a c}(E), \quad Q_{0} \nvdash_{\mathscr{C}_{0, \mathrm{ac}}}=\int Q_{0}(E) d P_{\mathrm{ac}}(E) .
$$

Here the so-called on-shell $S$-matrix $S\left(E ; H, H_{0}\right)$ is a unitary operator on the Hilbert space $\mathscr{H}(E)$ corresponding to the energy value $E \geqq 0$ (formally $\left.\mathscr{H}(E)=\frac{d P_{\mathrm{ac}}(E)}{d E} \mathscr{H}\right)$. Here and in what follows, we will always assume $E$ to be in $\sigma_{\mathrm{ac}}\left(H_{0}\right)$, the absolutely continuous spectrum of $H_{0}$. Since $Q_{0}^{2}=H_{0}$, we have

$$
Q_{0}(E)^{2}=E \mathbb{1}_{\mathscr{H}(E)},
$$

such that the s.a. operator $Q_{0}(E)$ on $\mathscr{H}(E)$ is bounded with bounded inverse if $E>0$, i.e. as long as we stay away from the threshold $E=0$.

Since $\tau$ commutes with $H_{0}$ we have a further decomposition $\mathscr{H}(E)$ $=\mathscr{H}_{+}(E) \oplus \mathscr{H}_{-}(E)$ into bosonic and fermionic parts. With respect to this decomposition we may write $Q_{0}(E)$ in matrix form

$$
Q_{0}(E)=\left(\begin{array}{cc}
0 & q_{-}(E) \\
q_{+}(E) & 0
\end{array}\right)
$$

Here $q_{+}(E)$ is an operator from $\mathscr{H}_{+}(E)$ to $\mathscr{H}_{-}(E)$ and $q_{-}(E)$ is an operator from $\mathscr{H}_{-}(E)$ to $\mathscr{H}_{+}(E)$. Relation $(2.10)$ now takes the form

$$
q_{-}(E) q_{+}(E)=E \mathbb{1}_{\mathscr{H}_{+}(E)}, \quad q_{+}(E) q_{-}(E)=E \mathbb{1}_{\mathscr{H}_{-}(E)} .
$$

From the boundedness of $Q_{0}(E)$ it follows that $q_{+}(E)$ and $q_{-}(E)$ are bounded. They have bounded inverses for $E>0$, since from (2.10) we conclude

$$
q_{+}(E)^{-1}=E^{-1} q_{-}(E), \quad q_{-}(E)^{-1}=E^{-1} q_{+}(E) .
$$

Note that due to (2.13) and the selfadjointness of $Q_{0}(E)$ the operator $P_{+}(E):=E^{-1 / 2} q_{+}(E)$ is a unitary operator from $\mathscr{H}_{+}(E)$ to $\mathscr{H}_{-}(E)$. Since $\tau$ also commutes with $S\left(H, H_{0}\right)$, we have the following on-shell decomposition:

$$
S\left(E ; H, H_{0}\right)=S_{+}\left(E ; H, H_{0}\right) \oplus S_{-}\left(E ; H, H_{0}\right),
$$

i.e. the on-shell $S$-matrix decomposes into a bosonic and a fermionic part. Next the relation

$$
Q_{0}(E) S\left(E ; H, H_{0}\right)=S\left(E ; H, H_{0}\right) Q_{0}(E)
$$

is a consequence of condition (c) in Definition 2.5. By (2.11) we rewrite this as

$$
q_{+}(E) S_{+}\left(E ; H, H_{0}\right) q_{+}(E)^{-1}=P_{+}(E) S_{+}\left(E, H, H_{0}\right) P_{+}(E)^{-1}=S_{-}\left(E ; H, H_{0}\right)
$$

for $E>0$. This proves the 
Proposition 2.7. Above threshold, for any $E$ in the absolutely continuous spectrum of $H_{0}$, the bosonic and fermionic on-shell $S$-matrices are unitarily equivalent.

We turn to a discussion of the total phase shifts in the bosonic and fermionic sectors at energy $E \geqq 0$. If they exist, they satisfy

$$
\exp 2 i \delta_{ \pm}\left(E ; H, H_{0}\right)=\operatorname{det} S_{ \pm}\left(E ; H, H_{0}\right) .
$$

These phase shifts are only defined modulo $\pi \mathbb{Z}$. In applications we have in mind, however, a unique choice can be made using the theory of the spectral shift function (see below). In particular we will make the convention that $\delta_{ \pm}\left(E, H_{0}, H_{0}\right)=0$. We therefore have the result

Proposition 2.8. The bosonic phase shift above threshold exists if and only if the fermionic phase shift exists such that

$$
\frac{1}{\pi}\left(\delta_{+}\left(E ; H, H_{0}\right)-\delta_{-}\left(E, H, H_{0}\right)\right)=n\left(E ; H, H_{0}\right) \in \mathbb{Z} .
$$

Also if they exist the supersymmetric determinant of the total on-shell S-matrix is equal to 1 :

$$
\operatorname{det}_{\tau} S\left(E ; H, H_{0}\right):=\frac{\operatorname{det} S_{+}\left(E ; H, H_{0}\right)}{\operatorname{det} S_{-}\left(E ; H, H_{0}\right)}=1 .
$$

Relation (2.18) has interesting consequences. A first simple result is

Proposition 2.9. Assume the absolutely continuous spectrum of $H_{0}$ (and hence of $H$ also $)$ is a connected subset of $\mathbb{R}^{+}$such that $\delta_{ \pm}\left(E ; H, H_{0}\right)$ exist and their difference is continuous in $E$ on this set. Then there is an integer, called the supersymmetric scattering index of $H$ and $H_{0}$ and denoted by $n\left(H, H_{0}\right)$, such that

$$
\frac{1}{\pi}\left(\delta_{+}\left(E ; H, H_{0}\right)-\delta_{-}\left(E ; H, H_{0}\right)\right)=n\left(H, H_{0}\right)
$$

for all $E$ in the absolutely continuous spectrum of $H_{0}$.

The very definition of $n\left(H, H_{0}\right)$ leads to two ways of determining this quantity from phase shift data in case the absolute continuous spectrum of $H_{0}$ is all of $\mathbb{R}^{+}$, the set of all nonnegative reals. One way is to look at the threshold behaviour $E \rightarrow 0$ of $\delta_{+}\left(E ; H, H_{0}\right)$ and $\delta_{-}\left(E ; H, H_{0}\right)$. The other one is to look at the high energy behaviour $E \rightarrow \infty$, where partial results are known [33, 32, 40].

The next result is a stability statement

Proposition 2.10. Let $H(\lambda)$ be a one-parameter family of Hamiltonians $(\lambda \in I, I$ an interval) with supersymmetry relative to a fixed $\tau$. Assume that for each $\lambda \in I$ the assumptions of Proposition 2.9 are fullfilled for $H=H(\lambda)$ and that in addition $\delta_{+}\left(E ; H(\lambda), H_{0}\right)-\delta_{-}\left(E, H(\lambda), H_{0}\right)$ is continuous in $\lambda$ for fixed $E$. Then $n\left(H(\lambda), H_{0}\right)$ is a constant on I. In particular, if there is $\lambda_{0} \in I$ such that $H\left(\lambda_{0}\right)=H_{0}$, then $n\left(H(\lambda), H_{0}\right)$ is identically zero on $I$. 
We now consider situations which guarantee the existence of $\delta_{ \pm}\left(E ; H, H_{0}\right)$, and a supersymmetric scattering theory, namely we assume that (compare Lemma 2.6)

$$
\left.\begin{array}{l}
\exp -t H-\exp t H_{0} \\
Q \exp -t H-Q_{0} \exp -t H_{0}
\end{array}\right\} \text { are of trace class for all } t>0 .
$$

Note that in general none of the conditions (2.21) is a consequence of the other. Then by the Krein theory of spectral shift functions (see e.g. [9]) there is a unique locally integrable function $\xi\left(E ; H, H_{0}\right)$ in $E$ such that $\xi\left(E ; H, H_{0}\right) e^{-t E}$ is integrable in $[0, \infty)$ with

$$
\text { Trace }\left(\exp -t H-\exp -t H_{0}\right)=-t \int_{0}^{\infty} \xi\left(E ; H, H_{0}\right) e^{-t E} d E .
$$

Indeed, the function

$$
\xi_{t}(\lambda)=\pi^{-1} \lim _{\varepsilon \rightarrow 0^{+}} \arg \operatorname{det}\left(\mathbb{1}+\left(e^{-t H}-e^{-t H_{0}}\right)\left(e^{-t H_{0}}-\lambda-i \varepsilon\right)^{-1}\right)
$$

is an integrable function with support in $[0,1]$ such that

$$
\text { Trace }\left(\exp -t H-\exp -t H_{0}\right)=\int_{0}^{1} \xi_{t}(\lambda) d \lambda .
$$

Note that the right-hand side of (2.23) is uniquely defined by the condition that it should tend to zero for $\operatorname{Im} \lambda \rightarrow+\infty$.

It is easy to show (compare e.g. [33, Lemma 3.1]) that the function $-\xi_{t}\left(e^{-t E}\right)$ in $E>0$ is independent of $t>0$. We denote this function by $\xi\left(E ; H, H_{0}\right)$ and obtain (2.22). Furthermore the phase-shift function $\delta\left(E ; H, H_{0}\right)$ then also exists almost everywhere on the absolute continuous spectrum of $H_{0}$ and may be identified with $-\pi \xi\left(E ; H, H_{0}\right)$ there (see e.g. [9]). This leads to a unique choice of phase shifts referred to above such that in particular $\delta\left(E ; H_{0}, H_{0}\right)=0$. In the applications we have in mind, the absolute continuous spectrum $\sigma_{\mathrm{ac}}\left(H_{0}\right)$ of $H_{0}$ will be all of $\mathbb{R}^{+}$so that in these cases we have

$$
\operatorname{Trace}\left(\exp -t H-\exp -t H_{0}\right)=\frac{t}{\pi} \int_{0}^{\infty} \delta\left(E ; H, H_{0}\right) e^{-t E} d E .
$$

Furthermore exp- $t H-$ exp- $t H_{0}$ restricted to $\mathscr{H}_{+}$and to $\mathscr{H}_{-}$respectively is also of trace class resulting in corresponding quantities $\xi_{ \pm}$and $\delta_{ \pm}$with similar relations. But then we have, using

$$
\begin{gathered}
\tau=\frac{1+\tau}{2}-\frac{(1-\tau)}{2}=P_{+}-P_{-}, \\
\text {Trace } \tau\left(\exp -t H-\exp -t H_{0}\right)=\frac{t}{\pi} \int_{0}^{\infty}\left(\delta_{+}\left(E ; H, H_{0}\right)-\delta_{-}\left(E ; H, H_{0}\right)\right) e^{-t E} d E \\
=t \int_{0}^{\infty} n\left(E ; H, H_{0}\right) e^{-t E} d E=n\left(H, H_{0}\right)
\end{gathered}
$$

whenever $n\left(E, H, H_{0}\right)=\delta_{+}\left(E, H, H_{0}\right)-\delta_{-}\left(E, H, H_{0}\right)$ is continuous in $E>0$. In general the spectral shift function will have integer valued discontinuities 
whenever $H$ or $H_{0}$ have a point spectrum, making $\delta_{+}$and $\delta_{-}$discontinuous. In certain cases it is possible to control these discontinuities. More precisely, let $P^{\text {point }}\left(H_{0}\right)$ and $P^{\text {point }}\left(H_{0}\right)$ be the projections onto the subspaces for the point spectrum of $H$ and $H_{0}$ respectively, such that

$$
\mathbb{1}-P^{\text {point }}(H)=P_{c}(H) \quad \text { and } \quad \mathbb{1}-P^{\text {point }}\left(H_{0}\right)=P_{c}\left(H_{0}\right)
$$

are the projections onto the continuous subspaces of $H$ and $H_{0}$ respectively. We now introduce the additional conditions

$$
\left.\begin{array}{l}
P^{\text {point }}(H) \exp -t H \\
P^{\text {point }}\left(H_{0}\right) \text { exp- } t H_{0}
\end{array}\right\} \text { are trace class for } t>0 .
$$

Then conditions (2.21a) and (2.27) guarantee the existence of the spectral shift functions $\xi^{c}\left(E ; H, H_{0}\right)$ and $n(E ; H)$ and $n\left(E, H_{0}\right)$ such that

$$
\begin{gathered}
\operatorname{Trace}\left(P_{c}(H) \exp -t H-P_{c}\left(H_{0}\right) \exp -t H_{0}\right)=t \int_{0}^{\infty} \xi^{c}\left(E ; H, H_{0}\right) e^{-t E} d E, \\
\text { Trace } P^{\text {point }}(H) \exp -t H=t \int_{0}^{\infty} n(E ; H) e^{-t E} d E, \\
\text { Trace } P^{\text {point }}\left(H_{0}\right) \exp -t H_{0}=t \int_{0}^{\infty} n\left(E ; H_{0}\right) e^{-t E} d E,
\end{gathered}
$$

and such that for almost all $E>0$,

$$
\xi\left(E ; H, H_{0}\right)=\xi^{c}\left(E ; H, H_{0}\right)+n(E ; H)-n\left(E, H_{0}\right) .
$$

A routine calculation shows that $n(E ; H)$ is the number of point eigenvalues of $H$ less or equal to $E$. An analogous statement holds for $n\left(E ; H_{0}\right)$.

The same discussion pertains to the bosonic and fermionic sector separately, where we denote the corresponding quantities by $\xi_{ \pm}^{c}\left(E ; H, H_{0}\right), n_{ \pm}(E ; H)$, and $n_{ \pm}\left(E ; H_{0}\right)$. Note that $\xi_{ \pm}=\xi_{ \pm}^{c}$, if the point spectrum for $H$ and $H_{0}$ is empty.

By the same arguments, which led us to Lemma 2.4, we have

Lemma 2.11. If the conditions (2.27) are satisfied, then there are integers $n^{\text {point }}(H)$ and $n^{\text {point }}\left(H_{0}\right)$ such that for all $E>0$,

$$
n_{+}(E ; H)-n_{-}(E ; H)=n^{\text {point }}(H), \quad n_{-}\left(E ; H_{0}\right)-n_{-}\left(E ; H_{0}\right)=n^{\text {point }}\left(H_{0}\right) .
$$

Note that if $H$ has purely discrete spectrum, such that exp- $t H$ is of trace class, then $n^{\text {point }}(H)$ equals the quantity in (2.3). In the presence of discrete eigenvalues we make a modified choice of the phase shift. Namely we let $\delta^{c}\left(E ; H, H_{0}\right)$ $=-\pi \xi^{c}\left(E ; H, H_{0}\right)$. Note that $\pi^{-1}\left(\delta-\delta^{c}\right)$ is always an integer, so both $\delta$ and $\delta^{c}$ are possible choices for a definition of the phase shift.

Now we are led to the following extension of Proposition 2.9.

Theorem 2.12. Let $\sigma_{\mathrm{ac}}\left(H_{0}\right)=\mathbb{R}^{+}$. Also assume the conditions (2.21) and (2.27) hold such that $\delta_{+}^{c}\left(E ; H, H_{0}\right)-\delta_{-}^{c}\left(E, H, H_{0}\right)$ is continuous in $E>0$. Then there is an integer $n^{c}\left(H, H_{0}\right)$ such that for all $E>0$,

$$
\frac{1}{\pi}\left(\delta_{+}^{c}\left(E ; H, H_{0}\right)-\delta_{-}^{c}\left(E ; H, H_{0}\right)\right)=n^{c}\left(H, H_{0}\right) .
$$


Also

$$
\operatorname{Trace} \tau\left(\exp -t H-\exp -t H_{0}\right)=n^{c}\left(H, H_{0}\right)+n^{\text {point }}(H)-n^{\text {point }}\left(H_{0}\right) .
$$

Here the last relation follows from the bosonic and fermionic version of (2.28)-(2.30) and Lemma 2.11 combined with (2.26). The condition $\sigma_{\mathrm{ac}}\left(H_{0}\right)=\mathbb{R}^{+}$is essential to obtain the $t$-independence of the left-hand side of (2.34) and (2.26) (see Theorem 2.14 below).

Remarks 2.13. (i) Note that the splitting off of discrete eigenvalues was only done in order to eliminate the discontinuities due to embedded eigenvalues in the continuum. Although we know of examples where $\delta^{c}$ still has discontinuities due to the appearance of resonances above threshold, we expect that in many applications $\delta_{ \pm}^{c}(E)$ will actually become continuous. Now all that is needed in the above arguments is the continuity of $\delta_{+}^{c}-\delta_{-}^{c}$ or equivalent of $\delta_{+}-\delta_{-}$in $E$. Hence if in fact the discontinuities of $\delta_{ \pm}^{c}$ are due to resonances above threshold, then we expect them to cancel in $\delta_{+}^{c}-\delta_{-}^{c}$ by supersymmetry in the same way as is the case for discrete eigenvalues. Therefore it would be interesting to find a general criterion which will ensure the continuity of $\delta_{ \pm}^{c}$ or their difference. When $E=0$ is the only element in the point spectrum, the above procedure is not necessary. In fact, this will be the case for the Laplace operator acting on differential forms on a Riemannian manifold without boundary, which is euclidean at infinity and which will be discussed in Sect. 4.

(ii) Nevertheless, the decomposition given by (2.34) is of general interest for the following reason. Although scattering theory gives sufficient conditions for $n\left(H, H_{0}\right)$ and $n^{c}\left(H, H_{0}\right)$ to exist and to be integers, in the cases we will discuss below, their explicit evaluation is either based on stability arguments (Proposition 2.10) or by methods not involving scattering states. However, we conjecture that $n^{c}\left(H, H_{0}\right)$ is calculable in terms of what physicists call zero energy resonances. In fact, in the cases where we have been able to check this, this conjecture held true. In this context it is worthwhile to point out the following obvious fact. Assume $H(\lambda)$ is a one parameter family of supersymmetric Hamiltonians such that $n\left(H(\lambda), H_{0}\right)$, $n^{c}\left(H(\lambda), H_{0}\right)$, and $n^{\text {point }}(H(\lambda))$ are all well defined. Then if two of these quantities are constant in $\lambda$, then so is the third.

(iii) In several publications on supersymmetric theories a so-called fractional Witten index has been studied (cf. [13] and references quoted there). This is connected with supersymmetric Hamiltonians whose energy spectrum has a continuous part. The regularized Witten index is then defined by giving a meaning to Trace $\left(\tau e^{-t H}\right)$ and then taking the limit $t \rightarrow \infty$. In most of the papers on fractional indices it is implicitly assumed that the Hilbert spaces of the bosonic and fermionic are identical. This in particular is the case for supersymmetric quantum mechanic models. All these models can be treated by our method.

(iv) Extended solutions in the sense of [7] may be considered as a special case of zero energy resonances. Now the dimension of extended solutions appear with a factor $1 / 2$ in certain index theorems [7]. It is a remarkable observation that such a factor $1 / 2$ also shows up in counting the contributions of resonances to the fractional Witten index.

We now elaborate on (iii) and (iv) of the preceding remark by giving a discussion of one model which has been considered by many authors. Let 
$\Phi \in C^{\infty}(\mathbb{R})$ with $\lim _{x \rightarrow \pm \infty} \Phi(x)=\Phi^{ \pm}$and set $L=d / d x+\Phi$ considered as an operator in $L^{2}(\mathbb{R})$ with domain $C_{c}^{\infty}(\mathbb{R})$. Let $Q$ be the unique selfadjoint extension of

$$
\left(\begin{array}{cc}
0 & L^{*} \\
L & 0
\end{array}\right)
$$

acting in $\mathscr{H}=L^{2}(\mathbb{R}) \oplus L^{2}(\mathbb{R})$. We set

$$
\tau=\left(\begin{array}{cc}
\mathbb{1} & 0 \\
0 & -\mathbb{1}
\end{array}\right)
$$

and $H_{ \pm}$(the restriction of $H=Q^{2}$ to the bosonic and fermionic section respectively) are the unique selfadjoint extensions of

$$
-\frac{d^{2}}{d x}+\Phi^{2} \mp \Phi^{\prime}
$$

acting on $C_{c}^{\infty}(\mathbb{R})$. For simplicity assume that $\Phi$ is constant outside a compact set. Now we define the free supercharge $Q_{0}$ as follows: Let $\mathbb{R}^{ \pm}$denote the positive and negative half-line respectively. Let $L_{0}^{ \pm}$be the closure of $d / d x+\Phi^{ \pm}$acting on $L^{2}\left(\mathbb{R}^{+}\right)$ with Dirichlet boundary conditions. Further, put

$$
Q_{0}^{ \pm}=\left(\begin{array}{cc}
0 & \left(L_{0}^{ \pm}\right)^{*} \\
L_{0}^{ \pm} & 0
\end{array}\right) .
$$

$Q_{0}^{ \pm}$is a selfadjoint operator in the Hilbert space $L^{2}\left(\mathbb{R}^{+}\right) \oplus L^{2}\left(\mathbb{R}^{+}\right)$. Now write

$$
\mathscr{H}=\left(L^{2}\left(\mathbb{R}^{-}\right) \oplus L^{2}\left(\mathbb{R}^{-}\right)\right) \oplus\left(L^{2}\left(\mathbb{R}^{+}\right) \oplus L^{2}\left(\mathbb{R}^{+}\right) .\right.
$$

With respect to this decomposition we may consider $Q_{0}=Q_{0}^{-}+Q_{0}^{+}$as a selfadjoint operator in $\mathscr{H} . Q_{0}$ is the free supercharge and $H_{0}=Q_{0}^{2}$ the free Hamiltonian. Now it is easy to see that exp- $t H-\exp -t H_{0}$ and $Q \exp -t H-Q_{0} \exp -t H_{0}$ are both trace class operators for all $t>0$. Therefore, our results can be applied to this situation. However, observe that the multiplicity of the continuous spectrum of $H$ and $H_{0}$ jumps if $\Phi^{+} \neq \Phi^{-}$. Therefore, the individual spectral shift functions $\xi_{ \pm}^{c}\left(E ; H, H_{0}\right)$ will not be continuous. But, using computations similar to those in [41], Chap. IX one can show that $\xi_{+}^{c}\left(E ; H, H_{0}\right)-\xi_{-}^{c}\left(E ; H, H_{0}\right)$ is a continuous function on $\mathbb{R}^{+}$. Furthermore, one has $n^{\text {point }}\left(H_{0}\right)=0$. Now, by Theorem 2.12 we get

$$
\left.n^{\text {point }}(H)=\operatorname{Trace} \tau\left(\exp -t H-\exp -t H_{0}\right)\right)-n^{c}\left(H, H_{0}\right) .
$$

By construction

$$
n^{\text {point }}(H)=\operatorname{dim} \operatorname{Ker} H^{+}-\operatorname{dim} \operatorname{Ker} H^{-} .
$$

(This is just $n_{B}^{E=0}-n_{F}^{E=0}$ in the notation of [52].) Analyzing the spectral shift function shows that $n^{c}\left(H, H_{0}\right)=0$ in all cases. Thus

$$
n^{\text {point }}(H)=\operatorname{Trace} \tau\left(\exp -t H-\exp -t H_{0}\right) \text {. }
$$

This demonstrates the advantage of our method. The right-hand side of (2.37) is independent of $t$ and gives the right answer. 
Finally the kernel of $H_{0}$ can be explicitly determined. We may write

$$
H_{0}=\left(\begin{array}{cc}
H_{0,+} & 0 \\
0 & H_{0,-}
\end{array}\right),
$$

where $H_{0,+}$ is given in $L^{2}\left(\mathbb{R}^{ \pm}\right)$as the selfadjoint extension of $-d^{2} / d x^{2}+\Phi^{ \pm 2}$ with Dirichlet boundary conditions and $H_{0,-}$ is given on $L^{2}\left(\mathbb{R}^{ \pm}\right)$as the selfadjoint extension of $-d^{2} / d x^{2}+\Phi^{ \pm 2}$ with respect to the boundary conditions

$$
\left.\left(-\frac{d}{d x}+\Phi^{ \pm}\right) f\right|_{x=0}=0 .
$$

The kernels of exp- $t H_{0,+}$ and exp- $t H_{0,-}$ can be explicitly described (cf. [7] p. 52). In particular exp- $t H_{0,+}-\exp -t H_{0,-}$ is of trace class. Thus we may rewrite (2.37) as

$$
\operatorname{Trace}\left(\exp -t H_{+}-\exp -t H_{-}\right)=n^{\mathrm{point}}(H)+\operatorname{Trace}\left(\exp -t H_{0,+}-\exp -t H_{0_{-}-}\right) \text {. }
$$

It is easy to determine

$$
\Delta_{0}=\lim _{t \rightarrow \infty} \operatorname{Trace}\left(\exp -t H_{0,+}-\exp -t H_{0,-}\right) .
$$

One has

$$
\Delta_{0}=\left\{\begin{array}{ccl}
1 / 2 & \text { if } & \Phi^{+}=0 \\
-1 / 2 & \text { if } & \Phi^{-}=0 \\
0 & \text { if } & \operatorname{sign} \Phi^{+}=\operatorname{sign} \Phi^{-} \\
1 & \text { if } & \operatorname{sign} \Phi^{+} \neq \operatorname{sign} \Phi^{-}
\end{array}\right.
$$

This coincides with the results obtained [13].

The next result relates our set-up more closely to standard index theorems. It also explains the role of the condition $\sigma_{\mathrm{ac}}\left(H_{0}\right)=\mathbb{R}^{+}$in Theorem 2.12. The conditions in the next theorem guarantee in particular that $Q$ and $Q_{0}$ are Fredholm operators such that the quantity

$$
\tau-\operatorname{Index} Q=\operatorname{dim} \operatorname{Ker}\left(Q \mid \mathscr{H}_{+}\right)-\operatorname{dim} \operatorname{Ker}\left(Q \mid \mathscr{H}_{-}\right)
$$

is well defined. We have $\tau$-Index $Q=n^{\text {point }}(H)$, whenever both these quantities are defined.

Theorem 2.14. Assume conditions (2.21) and (2.27) hold such that

$$
\sigma_{\mathrm{ac}}(H)=\sigma_{a c}\left(H_{0}\right)=\left[\varepsilon_{0}, \infty\right), \quad\left(\varepsilon_{0}>0\right)
$$

and the singular continuous spectra of $H_{0}$ and $H$ are empty. In addition let $\delta_{+}^{c}\left(E ; H, H_{0}\right)-\delta_{-}^{c}\left(E ; H, H_{0}\right)$ be continuous in $E$ on $\left[\varepsilon_{0}, \infty\right)$. Then for all $t>0$,

$$
\text { Trace } \tau\left(\exp -t H-\exp -t H_{0}\right)=\tau \text {-Index } Q-\tau \text {-Index } Q_{0}+n^{c}\left(H, H_{0}\right) e^{-\varepsilon_{0} t} \text {. }
$$

In particular

$$
\begin{gathered}
\lim _{t \rightarrow 0} \operatorname{Trace} \tau\left(\exp -t H-\exp -t H_{0}\right)=\tau \text {-Index } Q-\tau \text {-Index } Q_{0}+n^{c}\left(H, H_{0}\right), \\
\lim _{t \rightarrow \infty} \operatorname{Trace} \tau\left(\exp -t H-\exp -t H_{0}\right)=\tau-\operatorname{Index} Q-\tau-\operatorname{Index} Q_{0}
\end{gathered}
$$


The proof is an easy adaptation of the arguments leading to Theorem 2.12. In fact note that $\xi_{ \pm}^{c}\left(E ; H, H_{0}\right)$ has support in $\left[\varepsilon_{0}, \infty\right)$ due to the absence of a singular continuous spectrum and may therefore be identified with $-\frac{1}{\pi} \delta_{ \pm}^{c}\left(E ; H, H_{0}\right)$. Again $n^{c}\left(H, H_{0}\right)$ is defined by relation (2.33) now valid for $E \in\left[\varepsilon_{0}, \infty\right)$. Thus

$$
\operatorname{Trace} \tau\left(\exp -t H-\exp -t H_{0}\right)=n^{\mathrm{point}}(H)-n^{\mathrm{point}}\left(H_{0}\right)+t \int_{\varepsilon_{0}}^{\infty} n^{c}\left(H, H_{0}\right) e^{-t E} d E
$$

and (2.42) follows.

In view of Lemma 2.4, the quantity (2.34) may also be viewed as a relative index in the sense of [31]. This will become clearer when we discuss scattering theory by obstacles and give a topological interpretation of the resulting supersymmetric scattering index. In this context, the following chain rule is of interest. Instead of considering the pair $\left(H, H_{0}\right)$ in Theorem 2.12, we consider two supersymmetric pairs $\left(H_{2}, H_{1}\right)$ and $\left(H_{1}, H_{0}\right)\left(H_{i}=Q_{i}^{2}, i=0,1,2\right)$ with a fixed involution.

Proposition 2.15. Assume the conditions of Theorem 2.12 hold for the pair $\left(\mathrm{H}_{2}, \mathrm{H}_{1}\right)$ as well as for the pair $\left(H_{1}, H_{0}\right)$. Then the same conditions hold for the pair $\left(H_{2}, H_{0}\right)$ such that

$$
n^{c}\left(H_{2}, H_{0}\right)=n^{c}\left(H_{2}, H_{1}\right)+n^{c}\left(H_{1}, H_{0}\right) .
$$

In particular $n^{c}\left(H_{1}, H_{0}\right)$ is skew in $H_{1}$ and $H_{0}$ :

$$
n^{c}\left(H_{1}, H_{0}\right)=-n^{c}\left(H_{0}, H_{1}\right) \text {. }
$$

Proof. That $\delta_{ \pm}^{c}\left(E ; H_{2}, H_{0}\right)$ is continuous in $E>0$ follows from the chain rule

$$
\delta_{ \pm}^{c}\left(E ; H_{2}, H_{0}\right)=\delta_{ \pm}^{c}\left(E ; H_{2}, H_{1}\right)+\delta_{ \pm}^{c}\left(E ; H_{1}, H_{0}\right),
$$

which is a consequence of the uniqueness of the spectral shift function. Alternatively this chain rule follows from the chain rule for Møller operators, see e.g. [46]. Relation (2.45) is then a trivial consequence of (2.26). q.e.d.

Assume now that the condition (2.21a) is replaced by the stronger condition that

$$
(H+z \mathbb{1})^{-\ell}-\left(H_{0}+z \mathbb{1}\right)^{-\ell} \quad(\operatorname{Re} z>0)
$$

is trace class for some $\ell>0$. Then under otherwise unchanged conditions we may give an alternative representation to (2.34). Indeed, by standard Laplace transformation arguments (see e.g. rel. 3.33 below)

$$
\operatorname{Trace} \tau\left\{\frac{z^{\ell}}{(H+z \mathbb{1})^{\ell}}-\frac{z^{\ell}}{\left(H_{0}+z \mathbb{1}\right)^{\ell}}\right\}=n^{c}\left(H, H_{0}\right)+n^{\text {point }}(H)-n^{\text {point }}\left(H_{0}\right) \text {. }
$$

This formula is closely related to expressions appearing in e.g. [18] and [48], where the term containing $H_{0}$ does not appear. In fact, in the cases we will discuss, the local density of the term involving $H_{0}$ on the left-hand side of (2.34) or (2.46) has vanishing supertrace (see Remark 2.19 below).

We now give some concrete examples for a supersymmetric scattering theory, where the supercharge $Q$ is a smooth perturbation of $Q_{0}$. No proofs will be given, 
since similar results will be derived in the next sections for more complicated situations.

Example 2.16. Let $\mathscr{H}_{0}$ be the Hilbert space of complex square integrable forms on $\mathbb{R}^{N}$ (quantities with the subindex 0 pertain to the free theory). More precisely, let $*_{0}$ be the Hodge duality operator on forms given by the flat metric. On $\Omega_{c}\left(\mathbb{R}^{N}\right)$ consider the positive definite quadratic form given as

$$
\left(\varphi, \varphi^{\prime}\right)_{0}=\int_{\mathbb{R}^{N}} \bar{\varphi} \wedge *_{0} \varphi^{\prime}
$$

Then $\mathscr{H}_{0}$ is obtained by completion. We write $\mathscr{H}_{0}=\bigoplus_{p=0}^{N} \mathscr{H}_{0}^{p}$, where $\mathscr{H}_{0}^{p}$ is the closed subspace consisting of square integrable $p$-forms. We recall (see Example 2.2) that $P$ is the linear operator which is $p$ on $p$-forms. $*_{0}$ extends to a unitary operator on $\mathscr{H}_{0}$ mapping $\Omega_{c}\left(\mathbb{R}^{N}\right)$ into itself such that

$$
*_{0} \circ *_{0}=(-1)^{N(N-P)} \text {. }
$$

Let $d_{0}$ be the closure in $\mathscr{H}_{0}$ of the exterior derivative on $\Omega_{c}\left(\mathbb{R}^{N}\right)$. Similarly let $\delta_{0}=d_{0}^{*}$, the adjoint of $d_{0}$, be the closure of the coderivative on $\Omega_{c}\left(R^{N}\right)$ such that

$$
\delta_{0}=*_{0} \circ d_{0} \circ *_{0} \circ(-1)^{N+N P+1} .
$$

The operator $Q_{0}=d_{0}+\delta_{0}$ is selfadjoint and $H_{0}=Q_{0}^{2}$ is the Laplace operator $\Delta_{0}$ on $\mathbb{R}^{N}$. We denote by $H_{0}^{p}=\Delta_{0}^{p}$ its restriction to $\mathscr{H}_{0}^{p}$. For a form $\varphi$ written as

$$
\varphi=\sum_{p=0}^{N} \sum_{i_{1} \ldots i_{p}} \varphi_{i_{1} \ldots i_{p}}(x) d x^{i_{1}} \wedge \ldots \wedge d x^{i_{p}},
$$

we have for the resulting selfadjoint contraction semigroup $(t>0)$

$$
\left(\exp -t \Delta_{0} \varphi\right)_{i_{1} \ldots i_{p}}(x)=\left(\exp -t \Delta_{0}^{0} \varphi_{i_{1} \ldots i_{p}}\right)(x) \text {. }
$$

Obviously $\Delta_{0}$ has only absolutely continuous spectrum. We consider the following perturbation $Q$ of $Q_{0}$.

Let $\chi$ be a (for simplicity) real valued Schwartz function and consider the closed operator [53],

$$
d=e^{\chi} d_{0} e^{-\chi},
$$

and let $\delta$ be the adjoint $d^{*}$. Then $Q=d+\delta$ is selfadjoint and $H=Q^{2}$ is a smooth perturbation of $H_{0}$, having also only absolute continuous spectrum. Let $H^{p}$ denote the restriction of $H$ to $\mathscr{H}_{0}^{p}$. It has been shown in [3] that this pair $\left(Q, Q_{0}\right)$ defines a supersymmetric scattering theory. Furthermore by techniques used e.g. in [33] and [46] it may be shown that the phase shifts $\delta^{p}\left(E ; H, H_{0}\right)=\delta\left(E ; H^{p}, H_{0}^{p}\right)$ for the scattering theory in $\mathscr{H}_{0}^{p}$ are Hölder continuous of index $v<1 / 2$ for $E>0$ and that

$$
\delta^{p}\left(E ; H, H_{0}\right)(E+1)^{-\ell} \in L^{1}\left(\mathbb{R}^{+}, d E\right)
$$

whenever $\ell>N / 2+1$. Obviously

$$
\begin{aligned}
& \delta_{+}\left(E ; H, H_{0}\right)=\sum_{p \text { even }} \delta^{p}\left(E ; H, H_{0}\right), \\
& \delta_{-}\left(E ; H, H_{0}\right)=\sum_{p \text { odd }} \delta^{p}\left(E ; H, H_{0}\right) .
\end{aligned}
$$


Also the supersymmetric scattering index vanishes such that

$$
\sum_{p=0}^{N}(-1)^{p} \delta^{p}\left(E ; H, H_{0}\right)=0 \text { for } E>0 .
$$

Example 2.17. Let $g(x)=\left\{g_{i j}(x)\right\}_{1 \leq i, j \leq N}$ be a $C^{\infty}$ function $\mathbb{R}^{N}$ with values in the real positive definite $N \times N$ matrices such that $g_{i j}(x)-\delta_{i j}$ is in Schwartz space for all $i, j$. This defines a metric on $\mathbb{R}^{N}$, making it into an oriented Riemannian space. Let $\mathscr{H}$ denote the resulting Hilbert space of forms with the scalar product

$$
\left(\varphi, \varphi^{\prime}\right)=\int \bar{\varphi} \wedge * \varphi^{\prime},
$$

where $*$ is the Hodge operator obtained from $g$. Let now $d$ be the closure in $\mathscr{H}$ of the exterior derivative on $\Omega_{c}\left(\mathbb{R}^{N}\right) \subset \mathscr{H}$. With $\delta$ being the adjoint, the supercharge $Q$ is now $d+\delta$ such that $H=Q^{2}$ is the Laplace operator on this Riemannian manifold.

The present set-up is suited for scattering theory in two spaces [36] via the unitary intertwining operator $J^{-1}: \mathscr{H} \rightarrow \mathscr{H}_{0}$ given as follows: There is a symmetric matrix ${ }^{1 / 2} g(x)$ whose square is $g(x)$ and such that ${ }^{1 / 2} g_{i j}(x)-\delta_{i j}$ is in Schwartz space for all $i, j$. Then

with

$$
\left(J^{-1} \varphi\right)(x)=\sum_{p} \sum_{i_{1} \ldots i_{p}}\left(J^{-1} \varphi\right)_{i_{1} \ldots i_{p}}(x) d x^{i_{1}} \ldots d x^{i_{p}}
$$

$$
\left(J^{-1} \varphi\right)_{i_{1} \ldots i_{p}}(x)=\left(\operatorname{det} g_{i j}(x)\right)^{1 / 4} \sum_{j_{1}, \ldots, j_{p}} \prod_{\ell=1}^{p}{ }^{1 / 2} g^{i_{\ell}, j_{\ell}}(x) \varphi_{j_{1} \ldots j_{p}(x)},
$$

where ${ }^{1 / 2} g^{i j}(x)$ is the inverse of ${ }^{1 / 2} g_{j k}(x)$. Then again the phase shifts $\delta^{p}\left(E ; H, H_{0}, J\right)$ $=\delta^{p}\left(E ; J^{-1} H J, H_{0}\right)$ for $p$-forms are Hölder continuous of index $v<\frac{1}{2}$. Relation (2.53) holds and the supersymmetric scattering index vanishes giving (2.55) in this case too.

Example 2.18. Consider the set-up of the preceding two examples but now with a new involution. Assume $N$ is even and let $\tau_{0}$ be given as $\tau_{0}=*_{0} \circ i^{P(P-1)+N / 2}$ (see Example 2.2). This $\tau_{0}$ anticommutes with $Q$ and $Q_{0}$ as given in Example 2.16. To cover Example 2.17 we note that $Q$ defined there anticommutes with $\tau=* \circ i^{P(P-1)+N / 2}$ and that $J \tau_{0}=\tau J$ as required for a two Hilbert space supersymmetric scattering theory (see the remarks after Definition 2.5). Let now $\mathscr{H}_{0,+}$ and $\mathscr{H}_{0,-}$ denote the closed complementary subspaces consisting of selfdual and antiselfdual forms (with respect to $\tau_{0}$ ) in $\mathscr{H}_{0}$. We may apply the same analysis to the resulting phase shifts $\delta_{ \pm}\left(E ; H, H_{0}\right)$. Let us look at this in more detail.

Note that $\tau_{0}$ and $\tau$ induce unitary involutions in the spaces $\mathscr{H}_{0}^{N / 2}$ and $\mathscr{H}^{N / 2}$ of middle dimension respectively. Let $\delta_{ \pm}^{N / 2}\left(E ; H, H_{0}\right)$ denote the resulting phase shifts. Then since $*_{0}$ maps $p$-forms into $N-p$ forms

$$
\begin{aligned}
& \text { Trace } *_{0} \cdot i^{P(P-1)+N / 2}\left(\exp -t H-\exp -t H_{0}\right) \\
& =\text { Trace }_{\mathscr{H}^{\mathrm{N} / 2} *_{0}} \circ i^{\left(\frac{N}{2}\right)^{2}}\left(\exp -t H-\exp -t H_{0}\right)
\end{aligned}
$$

(where in the case of Example 2.17, $H$ has to be replaced by $J^{-1} H J$ ). This gives the general relation $(E>0)$

$$
\delta_{+}\left(E ; H, H_{0}\right)-\delta_{-}\left(E ; H, H_{0}\right)=\delta_{+}^{N / 2}\left(E ; H, H_{0}\right)-\delta_{-}^{N / 2}\left(E ; H, H_{0}\right)
$$


by the uniqueness of the Laplace transform. Next we distinguish the cases $N / 2$ even and $N / 2$ odd. If $N / 2$ is odd $\tau_{0}=*_{0} \circ i$ on $\mathscr{H}_{0}^{N / 2}$ and the right-hand side of (2.59) and hence of (2.60) vanishes. In fact, let $\varphi_{+}^{j}(j=1, \ldots)$ be a complete set of eigenforms of $\exp -t H-\exp -t H_{0}\left(\right.$ respectively exp- $t J^{-1} H J-\exp -t H_{0}$ in the case of Example 2.17) which are selfdual with respect to $\tau_{0}=*_{0} \circ i$. Then the complex conjugate form $\bar{\varphi}_{+}^{j}$ is also an eigenform with the same eigenvalue as $\varphi_{+}^{j}$. But $\bar{\varphi}_{+}^{j}$ is antiselfdual and the claim follows.

Therefore the only nontrivial case is when $N$ is divisible by 4 . Then the vanishing of (2.59) and (2.60) for the examples at hand, giving a vanishing supersymmetric scattering index, follows by a stability argument of the form of Proposition 2.10. Not surprisingly, for Example 2.17 this is just the statement that the relative signature index of the pair $\left(\mathbb{R}^{N}, g\right)$ and $\left(\mathbb{R}^{N}\right.$, flat metric) vanishes.

Remark 2.19. Observe that in the examples considered so far, the pointwise supertrace of the free heat kernel vanishes identically. In this sense, the free Hamiltonian acts only as a spectator.

\section{Obstacles}

Let $\mathcal{O}_{e} \subset \mathbb{R}^{N}$ be an exterior domain, in other words for, $N>1, \mathcal{O}_{e}$ is an open connected set such that $\mathcal{O}_{i}=\mathbb{R}^{N} \backslash \mathcal{O}_{e}$ is compact and for $N=1$ we require $\mathcal{O}_{i}$ to be a compact interval. $\mathcal{O}_{i}$ will be called an obstacle. We assume $\mathcal{O}_{e}$ to have a smooth boundary, i.e. the boundary $\partial \mathcal{O}_{e}$ is the set $\mathcal{O}_{i} \cap \overline{\mathcal{O}}_{e}$, supposed to be a smooth, compactly embedded manifold of dimension $N-1$. For $N>1, \mathcal{O}_{i}$ and hence also $\partial \mathcal{O}_{e}$ need not be connected. In $\mathscr{H}_{0}$, the space of square integrable forms on $\mathbb{R}^{N}$, let $\mathscr{H}_{e}$ and $\mathscr{H}_{i}$ denote the closed subspaces consisting of forms with support in $\mathcal{O}_{e}$ and $\mathcal{O}_{i}$ respectively. Obviously $\Omega_{c}\left(\mathcal{O}_{e}\right)$ and $\Omega\left(\mathcal{O}_{i}\right)$ are dense in $\mathscr{H}_{e}$ and $\mathscr{H}_{i}$ respectively and $\mathscr{H}_{0}=\mathscr{H}_{e} \oplus \mathscr{H}_{i}$. We recall from Sect. 2 that $d_{0}$ and $\delta_{0}=*_{0} \circ d \circ *_{0}(-1)^{N+N P+1}$ are the exterior derivative and coderivative on $\mathscr{H}_{0}$ respectively. Let $d$ and $\delta$ denote the closures in $\mathscr{H}_{e}$ of $d_{0}\left\lceil\Omega_{c}\left(\overline{\mathcal{O}}_{e}\right)\right.$ and $\delta_{0}\left\lceil\Omega_{c}\left(\overline{\mathcal{O}}_{e}\right)\right.$ respectively. Also let $d_{c}$ and $\delta_{c}$ denote the closures in $\mathscr{H}_{e}$ of $d_{0} \uparrow \Omega_{c}\left(\mathcal{O}_{e}\right)$ and $\delta_{0} \uparrow \Omega_{c}\left(\mathcal{O}_{e}\right)$ respectively. It is well known [28] that these closures exist and that

$$
d^{*}=\delta_{c}, \quad \delta^{*}=d_{c}, \quad d_{c}^{*}=\delta, \quad \delta_{c}^{*}=d
$$

in the sense of adjoint operators on $\mathscr{H}_{e}$. Also $d$ maps $\mathscr{D}(d)$, its domain of definition, into itself such that $d^{2}=0$. A similar relation holds for the other operators, i.e.

$$
d^{2}=\delta^{2}=d_{c}^{2}=\delta_{c}^{2}=0 \text {. }
$$

Among the four possible choices (see e.g. [20]) of resulting (nonnegative) Laplace operators with local boundary conditions, the following two selfadjoint operators (see [20]) have turned out to be of importance,

$$
\begin{gathered}
\Delta_{\mathrm{Abs}}=d \delta_{c}+\delta_{c} d=d d^{*}+d^{*} d, \\
\Delta_{\mathrm{Rel}}=d_{c} \delta+\delta d_{c}=\delta^{*} \delta+\delta \delta^{*} .
\end{gathered}
$$

They correspond to the so-called absolute and relative boundary conditions, the name being related to the fact that for compact Riemannian manifolds with 
boundary the corresponding spaces of harmonic forms are isomorphic to absolute and relative cohomology respectively. We will return to this point below. Obviously these two Laplace operators are dual to each other under $*_{0}$. The other two possible choices are the selfadjoint operator $d_{c} \delta+\delta_{c} d$ and the non-selfadjoint operator $d \delta_{c}+\delta d_{c}$. First we show how the requirement of supersymmetry in a natural way leads to the above two choices (3.3). In fact, we have the following

Theorem 3.1. The supersymmetric charges, which anticommute with $(-1)^{P}$,

$$
Q_{\mathrm{Abs}}=d+\delta_{c}=d+d^{*} \text { with } \mathscr{D}\left(Q_{\mathrm{Abs}}\right)=\mathscr{D}(d) \cap \mathscr{D}\left(\delta_{c}\right)
$$

and

$$
Q_{\mathrm{Rel}}=\delta+d_{c}=\delta+\delta^{*} \quad \text { with } \quad \mathscr{D}\left(Q_{\mathrm{Rel}}\right)=\mathscr{D}(\delta) \cap \mathscr{D}\left(d_{c}\right)
$$

are selfadjoint and their squares equal $\Delta_{\mathrm{Abs}}$ and $\Delta_{\mathrm{Rel}}$ respectively.

Remark. The same statements hold for

$$
\begin{gathered}
Q_{\mathrm{Abs}}^{\prime}=i\left(d-\delta_{c}\right)=i\left(d-d^{*}\right), \\
Q_{\mathrm{Rel}}^{\prime}=i\left(\delta-d_{c}\right)=i\left(\delta-\delta^{*}\right)
\end{gathered}
$$

as will become obvious from the proof.

Proof. We will prove the theorem for $Q_{\mathrm{Abs}}$ and $\Delta_{\mathrm{Abs}}$. The proof for $Q_{\mathrm{Rel}}$ and $\Delta_{\mathrm{Rel}}$ is similar or may be deduced from the duality under $*_{0}$. We first claim that $Q_{\mathrm{Abs}}$ is closed. To see this, assume there is a sequence $\varphi_{n}$ in $\mathscr{D}\left(Q_{\mathrm{Abs}}\right)$ such that $\varphi_{n}$ and $Q_{\mathrm{Abs}} \varphi_{n}$ are strongly convergent to vectors $\varphi$ and $\psi$ respectively. We have to show that $\varphi \in \mathscr{D}\left(Q_{\mathrm{Abs}}\right)$ and $\psi=Q_{\mathrm{Abs}} \varphi$. Now by definition of $\mathscr{D}\left(Q_{\mathrm{Abs}}\right) Q_{\mathrm{Abs}} \varphi_{n}=d \varphi_{n}+\delta_{c} \varphi_{n}$. Since $d$ is a closed operator, its kernel ker $d$ is a closed subspace, which by (3.1) and (3.2) is orthogonal to the image $\operatorname{Im} \delta_{c}$ of $\delta_{c}$. Hence kerd is also orthogonal to the closure $\overline{\operatorname{Im} \delta_{c}}$ of $\operatorname{Im} \delta_{c}$. This gives an orthogonal decomposition of $\mathscr{H}_{e}$ in the form $\mathscr{H}_{e}=\operatorname{ker} d \oplus \overline{\operatorname{Im} \delta_{c}} \oplus \operatorname{Remainder}$. Now $d \varphi_{n} \in \operatorname{ker} d$ and $\delta_{c} \varphi_{n} \in \operatorname{Im} \delta_{c}$. Since $Q_{\text {Abs }} \varphi_{n}$ is convergent by assumption, both $d \varphi_{n} \in \operatorname{ker} d$ and $\delta_{c} \varphi_{n} \in \overline{\operatorname{Im} \delta_{c}}$ have to be convergent. Call the limits $\psi^{1}$ and $\psi^{2}$ such that $\psi^{1}+\psi^{2}=\psi$. Since $d$ and $\delta_{c}$ are closed, we have $\varphi \in \mathscr{D}(d)$ and $\varphi \in \mathscr{D}\left(\delta_{c}\right)$ with $\psi^{1}=d \varphi$ and $\psi^{2}=\delta_{c} \varphi$. This shows that $Q_{\mathrm{Abs}}$ is closed.

Next we recall the definition of the Laplace operator $\Delta_{\mathrm{Abs}}$. It is the sum of the two operators $\delta_{c} d$ and $d \delta_{c}$ both of which are selfadjoint by von Neumanns theorem (see e.g. [37]).

In particular $\mathscr{D}\left(\Delta_{\mathrm{Abs}}\right) \subset \mathscr{D}\left(Q_{\mathrm{Abs}}\right)$ and $\mathscr{D}\left(\Delta_{\mathrm{Abs}}\right)$ consists of those vectors $\varphi$ in $\mathscr{D}\left(Q_{\mathrm{Abs}}\right)$ such that $d \varphi \in \mathscr{D}\left(\delta_{c}\right)$ and $\delta_{c} \varphi \in \mathscr{D}(d)$. Since also $d \varphi \in \mathscr{D}(d)$ and $\delta_{c} \varphi \in \mathscr{D}\left(\delta_{c}\right)$ we have that $\Delta_{\mathrm{Abs}} \subseteq Q_{\mathrm{Abs}}^{2}$. Because $\Delta_{\mathrm{Abs}}$ is selfadjoint and $Q_{\mathrm{Abs}}$ is symmetric, we have equality $\Delta_{\mathrm{Abs}}=Q_{\mathrm{Abs}}^{2}$. The theorem is therefore proved if we can show that $Q_{\mathrm{Abs}}$ is selfadjoint. This, however, is a trivial consequence of the elementary.

Lemma 3.2. Let $A$ be a closed, symmetric operator such that $A^{2}$ is selfadjoint. Then $A$ is selfadjoint.

Proof. It suffices to show that $A$ has vanishing defect indices (which are equal since $A$ is symmetric). So assume there is $\psi \neq 0$ such that for all $\varphi \in \mathscr{D}(A)$,

$$
(\psi, A \varphi)=i(\psi, \varphi) .
$$


Then for all $\varphi \in \mathscr{D}\left(A^{2}\right)$ we have

$$
\left(\psi, A^{2} \varphi\right)=i(\psi, A \varphi)=-(\psi, \varphi) .
$$

Since $A^{2}$ is selfadjoint, this implies $\psi \in \mathscr{D}\left(A^{2}\right)$ and $A^{2} \psi=-\psi$, contradicting the property that $A^{2}$ is nonnegative.

Next we prove the absence of point eigenvalues. We expect this result on exterior domains to be known. However, we were unable to locate a reference.

Proposition 3.3. The operators $\Delta_{\mathrm{Abs}}$ and $\Delta_{\mathrm{Rel}}$ have no point spectrum.

Proof. The proofs for $\Delta_{\mathrm{Abs}}$ and $\Delta_{\mathrm{Rel}}$ are identical, so we consider only the case $\Delta_{\mathrm{Abs}}$. Now assume there is an eigenvalue $\varepsilon \geqq 0$ with eigenform $\varphi$. The case $N=1$ is easy, since $\Delta_{\mathrm{Abs}}$ satisfies Neumann boundary conditions on the 0 -forms and Dirichlet boundary conditions on the 1 -forms. In both cases it is well known, that there are no discrete eigenvalues. Hence we may concentrate on the case $N \geqq 2$.

Since $\Delta_{\mathrm{Abs}}$ is elliptic, $\varphi$ is a $C^{\infty}$ form away from $\partial \mathcal{O}_{e}$ and each component $\varphi_{i_{1} \ldots i_{p}}(x)$ satisfies

$$
\left(-\sum_{j=1}^{N} \frac{\partial^{2}}{\partial x^{j 2}}-\varepsilon\right) \varphi_{i_{1} \ldots i_{p}}(x)=0 .
$$

Now consider a large open ball $B_{r_{0}}=\left\{|x|<r_{0}\right\}$ with $B_{r_{0}} \supseteqq \mathcal{O}_{i}$. On the complement of $B_{r_{0}}$ we introduce polar coordinates and we may view each $\varphi_{i_{1} \ldots i_{p}}(x)$ there as an element of

$$
L^{2}\left(\left[r_{0}, \infty\right), r^{N-1} d r\right) \otimes L^{2}\left(S^{N-1}, d \Omega\right),
$$

where $d \Omega$ is the canonical volume form on the $(N-1)$-sphere $S^{N-1}$. In polar coordinates we have

$$
-\sum_{j=1}^{N} \frac{\partial^{2}}{\partial x^{j 2}}=-\frac{\partial^{2}}{\partial r^{2}}-\frac{N-1}{r} \frac{\partial}{\partial r}+\frac{\tilde{\Delta}}{r^{2}},
$$

where $\tilde{\Delta}$ is the (positive) Laplacian of the sphere $S^{N-1}$. Let $\left\{Y_{n}(\Omega)\right\}$ be a complete orthonormal basis of eigenvectors (spherical functions) of $\widetilde{\Delta}$ with eigenvalues $\lambda_{n} \geqq 0$. Thus for fixed $i_{1} \ldots i_{p}$ we have the following expansion outside $B_{r_{0}}$.

$$
\varphi_{i_{1} \ldots i_{p}}(x)=\sum_{n} g_{n}(r) Y_{n}(\Omega),
$$

where due to (3.6) and (3.7) each $g_{n}(r)$ satisfies the differential equation

$$
\left(-\frac{d^{2}}{d r^{2}}-\frac{(N-1)}{r} \frac{d}{d r}+\frac{\lambda_{n}}{r^{2}}-\varepsilon\right) g_{n}(r)=0 \text {. }
$$

We now set

$$
h_{n}(r)=r^{\frac{N-1}{2}} g_{n}(r)
$$

such that $h_{n}(r) \in L^{2}\left(\left[r_{0}, \infty\right), d r\right)$ satisfies the Bessel differential equation

$$
\left(-\frac{d^{2}}{d r^{2}}+\frac{1}{4} \frac{(N-1)(N-3)}{r^{2}}-\frac{\lambda_{n}}{r^{2}}-\varepsilon\right) h_{n}(r)=0 \text {. }
$$


Now consider first the case $\varepsilon>0$. Then from the explicit form of the two fundamental solutions it follows that there is no square integrable solution $h_{n}(r)$. Thus we conclude that $\varphi_{i_{1} \ldots i_{p}}(x)=0$ outside $B_{r_{0}}$. By the uniqueness theorem for solutions of elliptic differential equations (see e.g. [4]) we have that $\varphi_{i_{1} \ldots i_{p}}(x)=0$ in $\mathcal{O}_{e}$, since $\mathcal{O}_{e}$ is connected by assumption.

We turn to the case $\varepsilon=0$, which is more delicate. First introduce $\ell_{n}$ by

$$
\ell_{n}\left(\ell_{n}+1\right)=\lambda_{n}+\frac{1}{4}(N-1)(N-3)
$$

such that Eq. (4.11) for $\varepsilon=0$ takes the form

$$
\left(-\frac{d^{2}}{d r^{2}}+\frac{\ell_{n}\left(\ell_{n}+1\right)}{r^{2}}\right) h_{n}(r)=0 .
$$

The two fundamental solutions to this equation are $r^{-\ell_{n}}$ and $r^{\ell_{n}+1}$ whenever $\ell_{n}>0$. In that case $h_{n}(r)$ has to be of the form $c_{n} r^{-\ell_{n}}$ to ensure square integrability with the additional restriction $\ell_{n}>1 / 2$. In the other cases, that is for the situation where $\ell_{n}$ is not larger than $1 / 2$ (such that $\lambda_{n}$ and $N$ are small), there is no square integrable solution. Thus (3.8) takes the form

$$
\varphi_{i_{1} \ldots i_{p}}(x)=\sum_{n, \ell_{n}>\frac{1}{2}} c_{n} r^{-\ell_{n}-\frac{N-1}{2}} Y_{n}(\Omega)
$$

with convergence in the $L^{2}$-sense. We want to prove pointwise convergence. For this purpose note that

$$
c_{n} r_{0}^{-\ell_{n}-\frac{N-1}{2}}=\int_{S^{N-1}} \varphi_{i_{1} \ldots i_{p}}\left(r_{0}, \Omega\right) Y_{n}(\Omega) d \Omega .
$$

By Schwarz' inequality this gives

$$
\left|c_{n}\right| \leqq r_{0}^{\ell_{n}+\frac{N-1}{2}}\left({ }_{s^{N-1}}\left|\varphi_{i_{1} \ldots i_{p}}\left(r_{0}, \Omega\right)\right|^{2} d \Omega\right)^{1 / 2} \leqq c\left(r_{0}\right) r_{0}^{\ell_{n}+\frac{N-1}{2}},
$$

since $\varphi_{i_{1} \ldots i_{p}}\left(r_{0}, \Omega\right)$ is a continuous and hence bounded function on the compact set $S^{N-1}$. This gives the a priori estimate for $\varphi_{i_{1} \ldots i_{p}}(x)$ :

$$
\begin{aligned}
& \left|\varphi_{i_{1} \ldots i_{p}}(x)\right| \leqq c\left(r_{0}\right) \sum_{n, \ell_{n}>\frac{1}{2}}\left(\frac{r_{0}}{r}\right)^{\ell_{n}+\frac{N-1}{2}}\left|Y_{n}(\Omega)\right| \\
& \quad \leqq c\left(r_{0}\right)\left(\sum_{n, \ell_{n}>\frac{1}{2}}\left(\frac{r_{0}}{r}\right)^{2 \ell_{n}+N-1}\left(\lambda_{n}+1\right)^{k}\right)^{1 / 2}\left(\sum_{n, \ell_{n}>\frac{1}{2}} \frac{\left|Y_{n}(\Omega)\right|^{2}}{\left(\lambda_{n}+1\right)^{k}}\right)^{1 / 2},
\end{aligned}
$$

where $k>0$ is arbitrary.

The second factor on the right-hand side equals the square root of $(\tilde{\Delta}+1)^{-k}(\Omega, \Omega)$, the kernel of the resolvent of $\tilde{\Delta}$ at 1 to the $k$-th power evaluated on the diagonal at $\Omega$. But for $k>N$ this is a continuous function on $S^{N-1}$ by standard Sobolev inequalities. Hence we have the a priori estimate

$$
\left|\varphi_{i_{1} \ldots i_{p}}(x)\right|^{2} \leqq c^{\prime}\left(r_{0}\right) \sum_{n, \ell_{n}>\frac{1}{2}}\left(\frac{r_{0}}{r}\right)^{2 \ell_{n}+N-1}\left(\lambda_{n}+1\right)^{k} .
$$


Now the eigenvalues $\lambda_{n}$ of $\tilde{\Delta}$ are of the form $(L+N-2) L$ with a multiplicity given by

$$
(2 L+N-2)(N+L-3) ![(N-2) ! L !]^{-1}
$$

(see e.g. [50, pp. 459 and 507]). Also for large $\lambda_{n}$ we have $\ell_{n} \sim \sqrt{\lambda_{n}}$. Hence the righthand side of (3.18) is convergent for any $r>r_{0}$ such that $\left|\varphi_{i_{1} \ldots i_{p}}(x)\right|^{2}$ tends to zero when $r=|x|$ tends to infinity. We use this result as follows. Let $|\varphi|^{2}(x)$ be the pointwise norm of the harmonic form $\varphi$. Choose $R>r_{0}$ so large that

$$
|\varphi|^{2}(\mathrm{x})<\sup _{y \in \partial \mathcal{O}_{e}}|\varphi|^{2}(y)
$$

for all $|x| \geqq R$. Next $|\varphi|^{2}(x)$ satisfies the inequality

$$
\sum_{j=1}^{N} \frac{\partial^{2}}{\partial x^{i 2}}|\varphi|^{2}(x) \geqq 0
$$

by Leibnitz rule since $\Delta_{\text {Abs }} \varphi=0$ by assumption. Also the function $|\varphi|^{2}(x)$ satisfies Neumann boundary conditions on $\partial \mathcal{O}_{e}$. To see this, let $x_{0} \in \partial \mathcal{O}_{e}$ and introduce a local coordinate system $\left\{\hat{x}^{i} \mid 1 \leqq i \leqq N\right\}$ at $x_{0}$ such that $\hat{x}^{N}$ is the outward normal coordinate, i.e. locally $\partial \mathcal{O}_{e}=\left(\hat{\bar{x}}^{N}\right)^{-1}(\{0\})$. With respect to this coordinate system, any $\varphi_{i_{1} \ldots i_{p}}(x)$ satisfies either $\varphi_{i_{1} \ldots i_{p}}\left(x_{0}\right)=0$ or $\frac{\partial}{\partial \hat{x}^{N}} \varphi_{i_{1} \ldots i_{p}}\left(x_{0}\right)=0$. Hence again by Leibniz rule

$$
\frac{\partial}{\partial \hat{\mathrm{X}}^{N}}|\varphi|^{2}\left(x_{0}\right)=0 .
$$

But now we arrive at a contradiction, if we assume $\varphi \neq 0$ to be a square integrable form. Indeed, for any $r \geqq R_{0},|\varphi|^{2}$ is a subharmonic function in the domain $D=B_{r} \cap \mathcal{O}_{e}$ and therefore attains its maximum on the boundary of $D$. Because of estimate (3.19), the maximum value must be attained on $\partial \mathcal{O}_{e}$ and the normal derivative has to be $<0$ ([27], p. 55). But this contradicts (3.21) and the proof of Proposition 3.3 is complete.

To abbreviate notation, in what follows let $\Delta_{e}$ stand for either $\Delta_{\mathrm{Abs}}$ or $\Delta_{\mathrm{Rel}}$. Similarly let $Q_{e}$ denote either $Q_{\mathrm{Abs}}$ or $Q_{\mathrm{Rel}}$. Also let $G_{t}(x, y),(x, y) \in \mathbb{R}^{N} \times \mathbb{R}^{N}$ stand for the kernel of exp- $t \Delta_{e} \oplus 0$ on $\mathscr{H}_{e} \oplus \mathscr{H}_{i}=\mathscr{H}_{0}$. Following Ray and Singer [45], we write this kernel as a double form. Thus

$$
G_{t}(x, y)=\sum_{p} \sum_{\substack{i_{1} \ldots i_{p} \\ j_{1} \ldots j_{p}}} G_{t, i_{1} \ldots i_{p} ; j_{1} \ldots j_{p}}(x, y) d x^{i_{1}} \wedge \ldots \wedge d x^{i_{p}} ; d y^{j_{1}} \wedge \ldots \wedge d y^{j_{p}}
$$

such that

$$
\left(\left(\exp -t \Delta_{e} \oplus 0\right) \varphi\right)(x)=\int_{\mathscr{O}_{e}} G_{t}(x, y) \wedge *_{0} \varphi(y),
$$

where the integration applies to the variable $y \in \mathcal{O}_{e}$. Here and in what follows, we will use the convention that

$$
\int_{N} A=\int_{N} A^{n}
$$


if $N$ is an $n$-dimensional submanifold and $A$ is a form, whose component of degree $n$ is $A^{n}$. Next let $G_{0 t}(x, y),(x, y) \in \mathbb{R}^{N} \times \mathbb{R}^{N}$ denote the kernel of $G_{0 t}=\exp -t \Delta_{0}$, $\Delta_{0}$ being as before Laplace operator on $\mathbb{R}^{N}$. Also let $Q_{0}=d_{0}+d_{0}^{*}$ be the associated supercharge such that $\Delta_{0}=Q_{0}^{2}$.

Introducing

$$
k(x, y, t)=(4 \pi t)^{-N / 2} \exp -\frac{(x-y)^{2}}{4 t}
$$

by $(2.43)$ we have

$$
G_{0 t}(x, y)=k(x, y, t) \sum_{p} \frac{1}{p !} \sum_{i_{1} \ldots i_{p}} d x^{i_{1}} \wedge \ldots d x^{i_{p}} ; d y^{i_{1}} \wedge \ldots d y^{i_{p}} .
$$

The construction of the kernel $G_{t}(x, y)$ via a Neumann series may be taken over from [45]. Only the situation where $(x, y) \in \mathcal{O}_{e} \times \mathcal{O}_{e}$ is relevant and then

$$
G_{t}(x, y)=\sum_{m=0}^{\infty}(-2)^{m} Q^{m}(x, y, t) .
$$

Here the $Q^{m}$ are defined recursively as

$$
Q^{0}(x, y, t)=G_{0 t}(x, y)
$$

and for $m \geqq 0$ with absolute boundary conditions

$$
\begin{aligned}
Q^{m+1}(x, y, t)= & -\int_{0}^{t} d t^{\prime} \int_{\partial \mathcal{G}_{e}}\left(\left\{\delta_{0} Q^{m}\left(u, y, t-t^{\prime}\right) \wedge *_{0} G_{0 t^{\prime}}(x, u)\right.\right. \\
& \left.+Q^{m}\left(u, y, t-t^{\prime}\right) \wedge *_{0} d_{0} G_{0 t^{\prime}}(x, u)\right)
\end{aligned}
$$

while for relative boundary conditions

$$
\begin{aligned}
Q^{m+1}(x, y, t)= & \int_{0}^{t} d t^{\prime} \int_{\partial \theta_{e}}\left(G_{0 t^{\prime}}(x, u) \wedge *_{0} d_{0} Q^{m}\left(u, y, t-t^{\prime}\right)\right. \\
& \left.+\delta_{0} G_{0 t^{\prime}}(x, u) \wedge *_{0} Q^{m}\left(u, y, t-t^{\prime}\right)\right) .
\end{aligned}
$$

Here all operations on the right are applied to the variable $u$ and $(x, y) \in \mathcal{O}_{e} \times \mathcal{O}_{e}$.

Let $D(x)$ for $x \in \mathcal{O}_{e}$ denote the distance from $\partial \mathcal{O}_{e}$. Then with the above choice for $r_{0}$ we have

$$
D(x) \geqq|x|-r_{0}
$$

uniformly in $x \in \mathcal{O}_{e}$.

Also for any double form $A(x, y)$ we set

$$
\|A(x, y)\|=\sup _{i_{1} \ldots i_{p}, j_{1} \ldots j_{p}}\left|A_{i_{1} \ldots i_{p} ; j_{1} \ldots j_{p}}(x, y)\right| .
$$

The discussion in [45] gives the following:

Lemma 3.4. For a given obstacle there is a finite $C>0$ such that for all $m \geqq 1, t>0$ and all $(x, y) \in \mathcal{O}_{e} \times \mathcal{O}_{e}$ the following estimates are valid.

$$
\left\|\left(\frac{\partial}{\partial x^{i}}\right)^{n} Q^{m}(x, y, t)\right\| \leqq C^{m}(\Gamma(m / 2))^{-1} t^{-n / 2} \exp \left(-\frac{1}{4 t}\left(D^{2}(x)+D^{2}(y)\right)\right) \begin{array}{r}
k(x, y, t), \\
n=0,1 .
\end{array}
$$


Let $M(\varepsilon)$ be the multiplication operator on $\mathscr{H}_{0}$ by $\exp \varepsilon|x|$. Estimate (3.30) gives the following result.

Corollary 3.5. For any $t>0$ the operators in $\mathscr{H}_{0}$

$$
\begin{gathered}
M\left(\frac{1}{8 t}\right)\left(\exp -t \Delta_{e} \oplus 0-\exp -t \Delta_{0}\right) M\left(\frac{1}{8 t}\right), \\
M\left(\frac{1}{8 t}\right)\left(\left(Q_{e}\right) \exp -t \Delta_{e} \oplus 0-\left(Q_{0}\right) \exp -t \Delta_{0}\right) M\left(\frac{1}{8 t}\right)
\end{gathered}
$$

are of trace class.

We will also need estimates on the kernels of powers $\left(\Delta_{e}+z \mathbb{1}\right)^{-\ell}(\operatorname{Re} z>0, \ell>0)$ of the resolvent of $\Delta_{e}$. We start with the familiar relation

$$
(H+z \mathbb{1})^{-\ell}=\Gamma(\ell)^{-1} \int_{0}^{\infty} e^{-t(H+z \mathbb{1})} t^{\ell-1} d t
$$

valid for any s.a. $H \geqq 0, \operatorname{Re} z>0$, and $\ell>0$ in the strong topology. The next estimates are derived from the above estimates on $G_{t}(x, y)$ by splitting the integral over $t$ into two parts, where the first goes from 0 to 1 .

Let

$$
D(x, y)= \begin{cases}\left(|x-y|^{2}+D^{2}(y)+D^{2}(x)\right)^{1 / 2} & \text { for }(x, y) \in \mathcal{O}_{e} \times \mathcal{O}_{e} \\ |x-y| & \text { otherwise }\end{cases}
$$

Lemma 3.6. For given obstacle and $\ell>0$ there is $C(\ell)<\infty$ such that

$$
\left\|\left(\left(\Delta_{e}+\mathbb{1}\right)^{-\ell} \oplus 0-\left(\Delta_{0}+\mathbb{1}\right)^{-\ell}\right)(x, y)\right\| \leqq C(\ell) D(x, y)^{-N+2 \ell} \exp -\frac{1}{2} D(x, y) .
$$

Corollary 3.7. For any $2 \ell>N+1$ the operator on $\mathscr{H}_{0}$

$$
\left(\Delta_{e}+\mathbb{1}\right)^{-\ell} \oplus 0-\left(\Delta_{0}+\mathbb{1}\right)^{-\ell}
$$

is trace class.

Although we actually will not need it, for completeness we also state the

Corollary 3.8. The operators $\Delta_{\mathrm{Abs}}$ and $\Delta_{\mathrm{Rel}}$ have no singular continuous spectrum.

Proof. We follow the lines of arguments given in [24, 22, and 44]. It is sufficient to prove that for $\psi$ in a dense set of $\mathscr{H}_{e}$, the strong limit

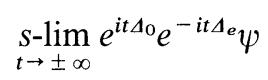

exists. Note that $\mathscr{H}_{e}$ is a subspace of $\mathscr{H}_{0}$. A sufficient condition is that

$$
\left(\Delta_{e}+\mathbb{1}\right)^{-1} \oplus 0-\left(\Delta_{0}+\mathbb{1}\right)^{-1}
$$

is compact and that the function $h(R)$ given as

$$
h(R)=\left\|\left(\left(\Delta_{e}+\mathbb{1}\right)^{-1} \oplus 0-\left(\Delta_{0}+\mathbb{1}\right)^{-1}\right) F(|x|>R)\right\|
$$


is integrable on $\mathbb{R}^{+}$, where $F(|x|>R)$ is the characteristic function of the set $\{|x|>R\}$. These two properties, however, are elementary consequences of estimate (3.35).

We now have all the ingredients to discuss the resulting scattering theory. Note first that estimates (3.31) and (3.32) are not quite conditions (2.21). This is due to the fact that $Q_{e}$ is an operator on $\mathscr{H}_{e} \subset \mathscr{H}_{0}$ only. To remedy this, let $\hat{Q}=Q_{e} \oplus 0$ with respect to the decomposition $\mathscr{H}_{0}=\mathscr{H}_{e} \oplus \mathscr{H}_{i}$, such that by $(3.32) \hat{Q} e^{-t \hat{Q}^{2}}-Q_{0} e^{-t Q_{0}^{2}}$ is of trace class. Then by Lemma 2.5 the Møller operators $W^{ \pm}\left(\hat{Q}, Q_{0}\right)$ exist. Since $\hat{Q}$ and $Q_{e}$ are identical on their absolutely continuous subspace, which is $\mathscr{H}_{e}$, the complete (two-space) Møller operators $W^{ \pm}\left(Q_{e}, Q_{0}, J\right)=\underset{t \rightarrow \pm \infty}{s-\lim _{\infty}} e^{i t Q_{e}} J e^{-i t Q_{0}}$ exist as maps from $\mathscr{H}_{0}$ to $\mathscr{H}_{e}$, where $J$ is the projection of $\mathscr{H}_{0}$ onto $\mathscr{H}_{e}$. By the invariance principle therefore also the Møller operators $W^{ \pm}\left(\Delta_{e}, \Delta_{0}, J\right)$ exist, defining a supersymmetric scattering theory. Now the arguments employed in [33] and [46] combined with Proposition 3.3 and Corollaries 3.5 and 3.7 give

Theorem 3.9. The scattering shifts

$$
\delta^{p}\left(E, \Delta_{e}, \Delta_{0}\right) \quad\left(\Delta_{e}=\Delta_{\mathrm{Abs}} \text { or } \Delta_{\mathrm{Re} 1}\right)
$$

for the scattering of p-forms exist, are real analytic and for any $\ell>N / 2+1$

$$
\delta^{p}\left(E ; \Delta_{e}, \Delta_{0}\right)(E+1)^{-\ell} \in L^{1}\left(\mathbb{R}^{+}, d E\right) .
$$

It remains to calculate the corresponding supersymmetric scattering index. Let $\chi(X)$ denote the (absolute) Euler characteristic of a compact manifold $X$ and $\chi(X, A)$ the (relative) Euler characteristic of the pair $X \supset A$, where $A$ is a compact submanifold (see e.g. [47, p. 205]).

Theorem 3.10. The supersymmetric scattering indices for scattering on an obstacle $\mathcal{O}_{i}$ are given as

$$
\begin{aligned}
& n\left(\Delta_{\mathrm{Abs}}, \Delta_{0}\right)=-\chi\left(\mathcal{O}_{i}, \partial \mathcal{O}_{i}\right), \\
& n\left(\Delta_{\mathrm{Rel}}, \Delta_{0}\right)=-\chi\left(\mathcal{O}_{i}\right) .
\end{aligned}
$$

Proof. Fix $R$ so that $\mathcal{O}_{i} \subseteq B_{R / 2}$. Let $M$ be a closed, compact Riemannian manifold so that $B_{3 R}$ is isometrically imbedded in $M$ and put $Y=M \backslash \mathcal{O}_{i}$. Let $G_{t}^{Y}(x, y)$ be the heat kernel for the Laplacian acting on $\Omega(Y)$ with absolute or relative boundary conditions on $\partial Y=\partial \mathcal{O}_{i}$. Following [7] on p. 54-55 we let $\varrho(a, b)(a, b \in \mathbb{R}, a<b)$ denote any increasing real valued $C^{\infty}$ function of the real variable $u$ such that $\varrho=0$ for $u \leqq a$ and $=1$ for $u \geqq b$. Then define four $C^{\infty}$ functions by $\Phi_{0}=\varrho(R / 2, R)$, $\Psi_{0}=\varrho(R, 3 / 2 R), \Phi_{1}=1-\varrho(3 / 2 R, 2 R), \Psi_{1}=1-\Psi_{0}$. As a parametrix for the fundamental solution for the heat equation for the operator $\Delta_{e}$ we may choose

$$
H_{t}(x, y)=\Psi_{0}\left(d\left(x, x_{0}\right)\right) G_{0 t}(x, y) \Phi_{0}\left(d\left(y, x_{0}\right)\right)+\Psi_{1}\left(d\left(x, x_{0}\right)\right) G_{t}^{Y}(x, y) \Phi_{1}\left(d\left(y, x_{0}\right)\right) .
$$

Here $x_{0}$ is the center of the ball $B_{3 R}$ and $d$ is the geodesic distance in $\mathbb{R}^{N}$. Starting with the parametrix $H_{t}$ we obtain the fundamental solution $G_{t}$ for the heat equation of $\Delta_{e}$ by the same construction as in $[7, \mathrm{p}$. 55]. The fundamental solution $G_{t}$ differs from the parametrix $H_{t}$ by an exponentially small term as $t \rightarrow 0$. Since we 
know that the supersymmetric scattering index is an integer, we may compute the supertrace by passing to the limit $t \rightarrow 0$. Therefore we can replace the fundamental solution $G_{t}$ by the parametrix $H_{t}$, and we get

$$
n\left(\Delta_{e}, \Delta_{0}\right)=\lim _{t \rightarrow 0} \int_{\mathscr{O}_{e}}\left(\operatorname{trace} H_{t+}(x, x)-\operatorname{trace} H_{t-}(x, x)\right) d x,
$$

where $H_{t+}$ and $H_{t-}$ denote the restrictions of $H_{t}$ to even and odd forms respectively and trace is the pointwise trace. Now observe that $\operatorname{trace} G_{0, t+}(x, x)$ $=\operatorname{trace} G_{0 t-}(x, x)$. Thus we are left with

$$
n\left(\Delta_{e}, \Delta_{0}\right)=\lim _{t \rightarrow 0} \int_{Y}\left(\operatorname{trace} G_{t+}^{Y}(x, x)-\operatorname{trace} G_{t-}^{Y}(x, x)\right) \Psi_{1}\left(d\left(x, x_{0}\right)\right) d x .
$$

The kernel $G_{t}^{Y}$ is constructed in [45]. It can be written as

$$
G_{t}^{Y}(x, y)=G(x, y, t)+Q(x, y, t),
$$

where $G$ is the restriction of the corresponding heat kernel on $M$ to $Y$ and $Q$ is the boundary correction term. Let $\mathscr{R}(x)$ be the Chern-Gauss-Bonnet density on $M$. By the local index theorem [6] we know that uniformly in $x$,

$$
\operatorname{trace} G_{+}(x, x, t)-\operatorname{trace} G_{-}(x, x, t)=\mathscr{R}(x)+0(t) .
$$

Now $\mathscr{R}$ vanishes on $B_{3 R} \subset M$ by construction. Hence the expression on the righthand side of (3.45) is equal to

$$
\lim _{t \rightarrow 0} \int_{Y}\left(\operatorname{trace} Q_{+}(x, x, t)-\operatorname{trace} Q_{-}(x, x, t)\right) \Psi_{1}\left(d\left(x, x_{0}\right)\right) d x .
$$

In view of formula (5.13) in [45] the asymptotic behaviour of $Q(x, x, t)$ as $t \rightarrow 0$ depends only on $x$ in an arbitrarily small neighborhood of the boundary $\partial Y$. Therefore we can delete the function $\Psi_{1}$ in (3.48). Using the local index theorem in [6] we see that (3.48) equals

$$
\chi(Y)-\int_{Y} \mathscr{R}(x)
$$

for absolute boundary conditions and

$$
\chi(Y, \partial Y)-\int_{Y} \mathscr{R}(x)
$$

for relative boundary conditions. Since $\mathscr{R}(x)=0$ on $B_{3 R}$ we can replace the integral over $Y$ by the integral over $M$. Employing the Chern-Gauss-Bonnet theorem for $M$ we obtain for $\left(3.49_{\mathrm{Abs}}\right)$ and $\left(3.49_{\mathrm{Rel}}\right)$ the expressions

$$
\chi(Y)-\chi(M)
$$

and

$$
\chi(Y, \partial Y)-\chi(M)
$$

respectively. Now observe that $M=Y \cup \mathcal{O}_{i}$. Therefore we may employ the formula (see e.g. [47, p. 205])

$$
\chi\left(X_{1}\right)+\chi\left(X_{2}\right)=\chi\left(X_{1} \cup X_{2}\right)+\chi\left(X_{1} \cap X_{2}\right) .
$$


Thus (3.49) finally equals

$$
\chi\left(\partial \mathcal{O}_{i}\right)-\chi\left(\mathcal{O}_{i}\right)=-\chi\left(\mathcal{O}_{i}, \partial \mathcal{O}_{i}\right)
$$

and

$$
-\chi\left(\mathcal{O}_{i}\right)
$$

respectively, concluding the proof of Theorem 3.10.

Using the Alexander duality (see e.g. [47, p. 298]) in the form $\chi\left(\mathcal{O}_{i}, \partial \mathcal{O}_{i}\right)$ $=(-1)^{N} \chi\left(\mathcal{O}_{i}\right)$, we can give Theorem 3.10 an alternative formulation

Corollary 3.11. The supersymmetric scattering indexes for scattering theory by an obstacle $\mathcal{O}_{i}$ are given as

$$
\begin{aligned}
n\left(\Delta_{\mathrm{Abs}}, \Delta_{0}\right) & = \begin{cases}1 / 2 \chi\left(\partial \mathcal{O}_{i}\right) & N \text { odd } \\
-\chi\left(\mathcal{O}_{i}\right) & N \text { even }\end{cases} \\
n\left(\Delta_{\mathrm{Re}}, \Delta_{0}\right) & = \begin{cases}-1 / 2 \chi\left(\partial \mathcal{O}_{i}\right) & N \text { odd } \\
-\chi\left(\mathcal{O}_{i}\right) & N \text { even } .\end{cases}
\end{aligned}
$$

There is yet another form in which we can state Theorem 3.10.

Let $\Delta_{i}$ be the Laplace operator on $\mathcal{O}_{i}$ with absolute or relative boundary conditions. Then again by the Hodge-de-Rham theorem for $\mathcal{O}_{i}$ [45], we have

Corollary 3.12. The following supertrace relation holds

$$
\text { Trace }(-1)^{P}\left(\operatorname{epx}-t\left(\Delta_{e} \oplus \Delta_{i}\right)-\exp -t \Delta_{0}\right)= \pm \chi\left(\mathcal{O}_{i}\right),
$$

where the plus sign holds for absolute boundary conditions and the minus sign for relative boundary conditions.

\section{Manifolds Euclidean at Infinity}

As another example of a supersymmetric scattering theory, in this section we will discuss the Gauss-Bonnet operator on manifolds which are euclidean at infinity. The extension to the asymptotically euclidean manifolds will be obtained in the next section by stability arguments.

Definition 4.1. A Riemannian manifold $\left(M^{N}, g\right)$ is euclidean at infinity if there is a compact submanifold $M_{i}^{N}$ such that $M^{N} \backslash M_{i}^{N}$ is a finite union of disjoint connected open sets $O_{j}(j=1 \ldots k)$, each of which is diffeomorphic under a map $\varphi_{j}$ to some $C B_{R_{j}}$. On each $O_{j}$ the metric is the pullback by $\varphi_{j}$ of the euclidean metric on $C B_{R_{j}}$.

Obviously manifolds which are euclidean at infinity are complete. In order not to burden our notation, we will assume that $k=1$, i.e. $M^{N} \backslash M_{i}^{N}$ has only one connected component which is isometrically isomorphic to some $C B_{R_{0}}$. Henceforth we will simply identify this set with $C B_{R_{0}}$. All our proofs, however, are easily extendable to general $k$. Therefore all results are formulated as to pertain to arbitrary $k$. By increasing $R_{0}$ and hence $M_{i}^{N}$ slightly, if necessary, we may assume $M^{N}$ to be flat in a neighborhood of $C B_{R_{0}}$. Let $\Delta=(d+\delta)^{2}$ be the selfadjoint Laplace operator acting in the Hilbert space $\mathscr{H}$ of square integrable forms on $\left(M^{N}, g\right)$. 
Standard arguments show that $d$ and $\delta$ defined on $\Omega_{c}\left(M^{N}\right)$ are closable operators with closure also denoted by $d$ and $\delta$ such that $d^{2}=\delta^{2}=0$ and $\mathscr{D}(d+\delta)=\mathscr{D}(d)$ $\cap \mathscr{D}(\delta)$. As a first result we have

Theorem 4.2. If $\left(M^{N}, g\right)$ is a Riemannian manifold without boundary which is euclidean at infinity, $\Delta$ has no point spectrum except possibly at the origin.

Indeed, our proof of the absence of positive eigenvalues in the case of obstacles may directly be carried over to our present situation.

To compare $\Delta$ with $\Delta_{0}$, the Laplace operator on the euclidean space $\mathbb{R}^{N}$, we introduce the Hilbert space

$$
\hat{\mathscr{H}}=\mathscr{H} \oplus \mathscr{H}^{<R_{0}},
$$

where we recall that $\mathscr{H}^{<R_{0}}$ is the Hilbert space of square integrable forms on $B_{R_{0}} \subset \mathbb{R}^{N}$. Alternatively we may write

$$
\hat{\mathscr{H}}=\mathscr{H}_{0} \oplus \mathscr{H}_{i},
$$

where $\mathscr{H}_{0}$ is the Hilbert space of square integrable forms on $\mathbb{R}^{N}$ and $\mathscr{H}_{i}$ is the Hilbert space of square integrable forms on $M_{i}^{N}$. In $\hat{\mathscr{H}}$ and for all $t>0$ introduce the operators $G_{t}=\exp -t \Delta \oplus 0$ with respect to the decomposition (4.1) and let $G_{t}^{\prime}=((d+\delta) \exp -t \Delta) \oplus 0$. Similarly let

$$
G_{0 t}=\exp -t \Delta_{0} \oplus 0 \quad \text { and } \quad G_{0 t}^{\prime}=\left(\left(d_{0}+\delta_{0}\right) \exp -t \Delta_{0}\right) \oplus 0
$$

with respect to the decomposition (4.2). Let $M(\varepsilon)$ be the multiplication operator $\exp \varepsilon|x|$ on $\mathscr{H}_{0}$ and let $\hat{M}(\varepsilon)$ be the operator on $\hat{\mathscr{H}}$ given as $M(\varepsilon) \oplus 0$ with respect to the decomposition (4.2). The next result again guarantees the existence of a supersymmetric scattering theory.

Theorem 4.3. The operators

$$
\hat{M}\left(\frac{1}{8 t}\right)\left(G_{t}-G_{0 t}\right) \hat{M}\left(\frac{1}{8 t}\right)
$$

and

$$
\hat{M}\left(\frac{1}{8 t}\right)\left(G_{t}^{\prime}-G_{0 t}^{\prime}\right) \hat{M}\left(\frac{1}{8 t}\right)
$$

are of trace class for all $t>0$.

Proof. The proof uses the same techniques already employed in the preceding section. Namely we introduce both on $M^{N}$ and on $\mathbb{R}^{N}$ absolute (say) boundary conditions on $\partial B_{R_{0}}$. Thus we obtain operators $\Delta_{\text {Abs }}$ in $\mathscr{H}$ and $\Delta_{0, \text { abs }}$ in $\mathscr{H}_{0}$. Now

$$
\Delta_{\mathrm{Abs}}=\Delta_{i \mathrm{Abs}} \oplus \Delta_{\mathrm{Abs}}^{>R_{0}}
$$

with respect to the decomposition

$$
\mathscr{H}=\mathscr{H}_{i} \oplus \mathscr{H}^{>R_{0}},
$$

where $\mathscr{H}^{>R_{0}}$ is the Hilbert space of square integrable forms on $C B_{R_{0}}$. Similarly we have

$$
\Delta_{0, \mathrm{Abs}}=\Delta_{\mathrm{Abs}}^{<R_{0}} \oplus \Delta_{\mathrm{Abs}}^{>R_{0}}
$$


with respect to the decomposition

$$
\mathscr{H}_{0}=\mathscr{H}^{<R_{0}} \oplus \mathscr{H}^{>R_{0}} .
$$

Now let $M^{\prime}(\varepsilon)$ be the multiplication operator on $\mathscr{H}$ given by $\exp \varepsilon|x| \cdot F\left(|x|>R_{0}\right)$. Here $F\left(|x|>R_{0}\right)$ is the characteristic function on $M^{N}$ of the set $C B_{R_{0}}$. Let $d_{\mathrm{Abs}}=d^{i}+d^{>R_{0}}$, where $d^{i}$ is the closure in $\mathscr{H}_{i}$ of the exterior derivative on $\Omega\left(M_{i}^{N}\right)$ and $d^{>R_{0}}$ is the closure in $\mathscr{H}^{>R_{0}}$ of the exterior derivative on $\Omega_{c}\left(C B_{R_{0}}\right)$, such that $\Delta_{\mathrm{Abs}}=\left(d_{\mathrm{Abs}}+d_{\mathrm{Abs}}^{*}\right)^{2} \cdot d_{0, \mathrm{Abs}}$ is defined in a similar fashion such that $\Delta_{0, \mathrm{Abs}}=\left(d_{0, \mathrm{Abs}}\right.$ $\left.+d_{0, \mathrm{Abs}}^{*}\right)^{2}$. By (4.5) and (4.7) it is sufficient to show that

$$
\begin{gathered}
M^{\prime}\left(\frac{1}{8 t}\right)\left(\exp -t \Delta-\exp -t \Delta_{\mathrm{Abs}}\right) M^{\prime}\left(\frac{1}{8 t}\right) \\
M^{\prime}\left(\frac{1}{8 t}\right)\left((d+\delta) \exp -t \Delta-\left(d_{\mathrm{Abs}}+d_{\mathrm{Abs}}^{*}\right) \exp -t \Delta_{\mathrm{Abs}}\right) M^{\prime}\left(\frac{1}{8 t}\right),
\end{gathered}
$$

and

$$
\begin{gathered}
M\left(\frac{1}{8 t}\right)\left(\exp -t \Delta_{0}-\exp -t \Delta_{0, \mathrm{Abs}}\right) M\left(\frac{1}{8 t}\right) \\
M\left(\frac{1}{8 t}\right)\left(\left(d_{0}+\delta_{0}\right) \exp -t \Delta_{0}-\left(d_{0, \mathrm{Abs}}+d_{0, \mathrm{Abs}}^{*}\right) \exp -t \Delta_{0, \mathrm{Abs}}\right) M\left(\frac{1}{8 t}\right)
\end{gathered}
$$

are of trace class for all $t>0$. These properties, however, may again be derived from the Ray-Singer contruction as in Corollary 3.5 .

Corollary 4.4. The operator $\Delta$ on $\mathscr{H}$ has no singular continuous spectrum.

This result is again obtained by an easy adaptation of the arguments used in the proof of Corollary 3.8.

Let now $\Delta^{p}$ be the restriction of $\Delta$ to $p$-forms. Then by Theorem 4.2 and by our by now standard arguments the spectral shift functions $\xi^{p}\left(E ; \Delta, \Delta_{0}\right)$ $:=\xi\left(E ; \Delta^{p}, \Delta_{0}^{p}\right)$ exist with the properties given by

Corollary 4.5. The spectral shift functions $\xi^{p}\left(E ; \Delta, \Delta_{0}\right)$ are real analytic in $E>0$ and satisfy

$$
\xi^{p}\left(E ; \Delta, \Delta_{0}\right)(E+1)^{-\ell} \in L^{1}\left(\mathbb{R}^{+}, d E\right)
$$

for any $\ell>N / 2+1$.

The last statement on the integrability is a consequence of explicit estimates on the kernels (4.9)-(4.12) which may be obtained analogously to the corresponding discussion in Sect. 3.

Again we identify the scattering phase shift functions $\delta^{p}\left(E ; \Delta, \Delta_{0}\right)$ with $-\pi \xi^{p}\left(E ; \Delta, \Delta_{0}\right)$ such that for all $t>0$ and $E>0$,

$$
\operatorname{Trace}(-1)^{P}\left(G_{t}-G_{0 t}\right)=\sum_{p=0}^{N}(-1)^{p} \frac{1}{\pi} \delta^{p}\left(E, \Delta, \Delta_{0}\right)=n\left(\Delta, \Delta_{0}\right) \in \mathbf{Z} .
$$

It remains to calculate $n\left(\Delta, \Delta_{0}\right)$. Now unlike in the preceding section, 0 will in general be in the point spectrum of $\Delta$ and by Theorem 2.12 it is of interest to 
calculate the contribution to (4.13) arising from the corresponding eigenforms, the so-called harmonic $L^{2}$-forms. More precisely, let $H_{(2)}^{p}$ be the closed subspace of $\mathscr{H}$ consisting of $p$-forms on $\left(M^{N}, g\right)$ which are in the kernel of the selfadjoint supercharge operator $d+\delta$. Then each element of $H_{(2)}^{p}$ is in particular in the kernel of $\Delta$. Conversely, if $\varphi \in \mathscr{H}$ is in the kernel of $\Delta$, then $0=(\Delta \varphi, \varphi)=\|(d+\delta) \varphi\|^{2}$ and hence $\varphi \in \bigoplus_{p=0}^{N} H_{(2)}^{p}$.

Let $\mathscr{R}(x)$ be the Chern-Gauss-Bonnet form on $\left(M^{N}, g\right)$.

Theorem 4.6. If $\left(M^{N}, g\right)$ is a Riemannian manifold without boundary, which is euclidean at infinity then the supersymmetric scattering index is given as

$$
n\left(\Delta, \Delta_{0}\right)=\int_{M^{N}} \mathscr{R}(x) .
$$

Furthermore, the space of harmonic $L^{2}$-forms is finite dimensional and

$$
n\left(\Delta, \Delta_{0}\right)=\sum_{p=0}^{N}(-1)^{p} \operatorname{dim} H_{(2)}^{p} .
$$

In terms of the notation employed in Theorem 2.12, this result just says that $n^{c}\left(\Delta, \Delta_{0}\right)$ vanishes. In other words, the index $n\left(\Delta, \Delta_{0}\right)$ has only contributions from the harmonic $L^{2}$-forms.

Denote the right-hand side of $(4.15)$ by $\chi_{(2)}(M)$, the $L^{2}$-Euler characteristic. Then our result is the noncompact version of the Chern-Gauss-Bonnet theorem saying that the $L^{2}$-Euler characteristic is the integral over the Chern-GaussBonnet form.

Proof of the First Part of Theorem 4.6. First we construct an approximate fundamental solution $H_{t}$ to the heat equation for the Laplacian $\Delta . H_{t}$ can be obtained from the heat kernel $G_{0 t}$ on $\mathbb{R}^{N}$ and the heat kernel $K_{t}$ for the Laplacian acting on the space of differential forms on a closed Riemannian manifold $X$ containing $M_{i}^{N}$ as an isometrically embedded submanifold.

Using again the construction of [7, p. 55] we glue $G_{0 t}$ to $K_{t}$ in a neighborhood of $\partial M_{i}^{N}$. Then $G_{t}-H_{t}$ is exponentially small. Hence in view of (4.13) we can replace $G_{t}$ by $H_{t}$. Employing the fact that

$$
\operatorname{trace}(-1)^{P} G_{0 t}(x, x)=0,
$$

we obtain

$$
n\left(\Delta, \Delta_{0}\right)=\lim _{t \rightarrow 0} \int_{\mathcal{M}^{N}} \operatorname{trace}(-1)^{P} H_{t}(x, x) d x .
$$

Now observe that by the local index theorem [6] one has trace $(-1)^{P} K_{t}(x, x)=\mathscr{R}(x)+0(t)$ uniformly in $x \in X$. Combined with (4.16) this gives the first part of Theorem 4.6.

To prove the second part of Theorem 4.6, we need some preparations. First we note that once the finite dimensionality of the space of harmonic $L^{2}$-forms is established, it suffices to consider the case when $N$ is even. In fact, if $N$ is odd, then by (4.14) $n\left(\Delta, \Delta_{0}\right)$ is zero. But now if $\varphi$ is an even harmonic $L^{2}$-form, then * $\varphi \neq 0$ is an odd harmonic $L^{2}$-form, so the right-hand side of (4.15) is also zero. 
Now observe that the right-hand side of (4.15) is just the $L^{2}$-index of the GaussBonnet operator, namely let $D_{G B}: \Omega_{+}(M) \rightarrow \Omega_{-}(M)$ be the restriction of $d+\delta$ to the space of even forms. Then the right-hand side of (4.15) is equal to $\operatorname{dim} \operatorname{Ker} D_{G B} \cap \mathscr{H}$ $-\operatorname{dim} \operatorname{Ker} D_{G B}^{*} \cap \mathscr{H}$. For the proof of relation (4.15) we shall transform this $L^{2}$-index problem into a nonlocal boundary value problem on $M_{i}^{N}$ in the sense of Atiyah, Patodi, and Singer and then apply Theorem 3.10 in [7]. We start by noting that $M^{N}$ can be written as $M_{i}^{N} \cup\left(\partial B_{R_{0}} \times\left[R_{0}, \infty\right)\right)$. In other words, $M^{N}$ is obtained from $M_{i}^{N}$ by attaching an infinite cylinder along the boundary. To study $d+\delta$ on the cylinder, i.e. as an operator on $\mathscr{H}^{>R_{0}} \mathrm{C} \mathscr{H}$, it is convenient to write $d+\delta$ in polar coordinates as done for example in [14]. For the convenience of the reader and to establish notation, we present a quick derivation. Let $\varphi$ be a smooth form with compact support in $\overline{C B}_{R_{0}}$. Then we may write

$$
\varphi=\varphi_{1}+d r \wedge \varphi_{2},
$$

where we view $\varphi_{j}(j=1,2)$ as smooth maps from $\left[R_{0}, \infty\right)$ into the space $\Omega\left(S^{N-1}\right)$. Let $\tilde{d}$ and $\tilde{\delta}$ denote the exterior derivative and the coderivative on $S^{N-1}$. Then obviously

$$
d \varphi=\tilde{d} \varphi+d r \wedge \frac{\partial \varphi_{1}}{\partial r}-d r \wedge \mathcal{d}_{\varphi_{2}}
$$

An easy argument shows that the norm of $\varphi$ in $\mathscr{H}^{>R_{0}}$ is given by

$$
\|\varphi\|^{2}=\sum_{j=1}^{2} \int_{R_{0}}^{\infty}\left\|r^{-P} \varphi_{j}\right\|_{\sim}^{2}(r) r^{N-1} d r,
$$

where \|\|$_{\sim}$ is the canonical Hilbert space norm for forms on $S^{N-1}$. It is therefore appropriate to introduce the quantities

$$
\psi_{j}(r)=r^{-P+\frac{N-1}{2}} \varphi_{j}(r) .
$$

Now for any $R_{0}-\varepsilon \leqq R<R^{\prime}$ the map $\varphi \mapsto\left(\psi_{1}, \psi_{2}\right)$ defines an isomorphism

$$
I\left(R, R^{\prime}\right): \Omega_{c}\left(\overline{C B}_{R} \backslash C B_{R^{\prime}}\right) \rightarrow C_{c}^{\infty}\left(\left[R, R^{\prime}\right], \Omega\left(S_{N-1}\right) \oplus \Omega\left(S_{N-1}\right)\right) .
$$

In particular for $I=I\left(R_{0}, \infty\right)$ and $I(\varphi)=\left(\psi_{1}, \psi_{2}\right)$ we have

$$
\|\varphi\|^{2}=\sum_{j=1}^{2} \int_{R_{0}}^{\infty}\left\|\psi_{j}\right\|_{\sim}^{2}(r) d r .
$$

Moreover one has

$$
I d I^{-1}=\left(\begin{array}{cc}
\frac{1}{r} \mathfrak{d} & 0 \\
\frac{1}{r}\left(P-\frac{N-1}{2}\right)+\frac{\partial}{\partial r} & -\frac{1}{r} \tilde{d}
\end{array}\right) .
$$

$I \delta I^{-1}$ is then simply the adjoint matrix. Thus we obtain the following matrix representation for $d+\delta$ acting on forms on $\partial B_{R_{0}} \times\left[R_{0}, \infty\right) \subset M^{N}$,

$$
I(d+\delta) I^{-1}=\sigma\left(\frac{\partial}{\partial r}+\frac{1}{r} A\right)
$$


with

and

$$
\sigma=\left(\begin{array}{cc}
0 & -\mathbb{1} \\
\mathbb{1} & 0
\end{array}\right)
$$

$$
A=\left(\begin{array}{cc}
P-\frac{N-1}{2} & -(\tilde{d}+\tilde{\delta}) \\
-(\tilde{d}+\tilde{\delta}) & -\left(P-\frac{N-1}{2}\right)
\end{array}\right)
$$

Since we are interested in the situation where $d+\delta$ acts on even forms, $\psi_{1}$ above is even and $\psi_{2}$ is odd. Note also that in this representation $\sigma$ defines on isomorphism from the even to the odd forms in $\mathscr{H}^{>R_{0}}$ with inverse $(-\sigma)$. This leads us to the following consideration. Let $\tilde{\mathscr{H}}$ denote the Hilbert space of square integrable forms on $S^{N-1}$. Decompose $\tilde{\psi} \in \tilde{\mathscr{H}}$ as $\tilde{\psi}_{1}-\tilde{\psi}_{2}$, where $\tilde{\psi}_{1}$ is even and $\tilde{\psi}_{2}$ is odd. With respect to this two-component presentation $\left(\tilde{\psi}_{1}, \tilde{\psi}_{2}\right)$ for $\tilde{\psi}, A$ corresponds to a selfadjoint operator $\tilde{A}$ on $\tilde{\mathscr{H}}$ given as

$$
\tilde{A}=C(P)+(\tilde{d}+\tilde{\delta})
$$

where

$$
C(P)=(-1)^{P}\left(P-\frac{(N-1)}{2}\right) .
$$

With these preparations we may now formulate the nonlocal boundary value problem appropriate for the situation at hand. Let $\widetilde{P}$ be the spectral projection of $\tilde{A}$ corresponding to eigenvalues $<0$ and let $M_{\varepsilon} \subset M_{i}^{N}$ be the $\varepsilon$-tubular neighborhood of $\partial M_{i}^{N}$. Given $\varphi$ in $\Omega_{+}\left(M_{i}^{N}\right)$ let $\psi_{1}$ and $\psi_{2}$ be the forms associated to $\varphi$ restricted to $M_{\varepsilon}$ via $I\left(R_{0}-\varepsilon, R_{0}\right)$. Let $\Omega_{+}\left(M_{i}^{N} ; \widetilde{P}\right) \subset \Omega_{+}\left(M_{i}^{N}\right)$ be the set of forms satisfying the following boundary condition

$$
\widetilde{P}\left(\left(\psi_{1}-\psi_{2}\right)\left(R_{0}\right)\right)=0 \text {. }
$$

Then

$$
D: \Omega_{+}\left(M_{i}^{N}, \widetilde{P}\right) \rightarrow \Omega_{-}\left(M_{i}^{N}\right)
$$

is defined to be $d+\delta$ restricted to $\Omega_{+}\left(M_{i}^{N}, \widetilde{P}\right)$.

$D^{*}$, the formal adjoint of $D$, is now an operator

$$
D^{*}: \Omega_{-}\left(M_{i}^{N}, 1-\widetilde{P}\right) \rightarrow \Omega_{+}\left(M_{i}^{N}\right),
$$

where $\Omega_{-}\left(M_{i}^{N}, 1-\widetilde{P}\right)$ is defined similar to $\Omega_{+}\left(M_{i}^{N}, \widetilde{P}\right)$ : If the odd form $\varphi$ corresponds to $\left(\psi_{1}, \psi_{2}\right)$ via $I\left(R_{0}-\varepsilon, R_{0}\right)$ then $\varphi \in \Omega_{-}\left(M_{i}^{N}, 1-\widetilde{P}\right)$ iff

$$
(1-\widetilde{P})\left(\left(\psi_{1}+\psi_{2}\right)\left(R_{0}\right)\right)=0 \text {. }
$$

Note that near the boundary $\partial M_{i}^{N}=\partial B_{R}, D^{*}$ takes the form

$$
D^{*}=\left(-\frac{\partial}{\partial r}+\frac{1}{r} A\right)(-\sigma)
$$

in the two-component representation $\left(\psi_{1}, \psi_{2}\right)$ of $\varphi$. 
We now have the following

Proposition 4.7. For $N \geqq 2$,

(i) $\operatorname{Ker} D$ is linearly isomorphic to the space of even harmonic $L^{2}$-forms on $M^{N}$,

(ii) $\operatorname{Ker} D^{*}$ is linearly isomorphic to the space of odd harmonic $L^{2}$-forms on $M^{N}$.

Before we give a proof of this proposition we turn to a

Proof of the Second Part of Theorem 4.6. Proposition 4.7 states in particular that the spaces $H_{(2)}^{p}$ are all finite dimensional, hence by a remark made above, it suffices to consider the case when $N$ is even. But again by Proposition 4.7,

$$
\operatorname{Index} D=\operatorname{dim} \operatorname{Ker} D-\operatorname{dim} \operatorname{Ker} D^{*}=\sum_{p=0}^{N}(-1)^{p} \operatorname{dim} H_{(2)}^{p},
$$

thus it suffices to calculate the index of $D$. Now Index $D$ may be written as a sum of three contributions (see e.g. [30] or [7, Theorem 3.10]). The first term is the integrated Chern-Gauss-Bonnet form of $M_{i}^{N}$. The second term is the $\eta$-invariant of $\tilde{A}$ and the third term is an integral over $\partial B_{R_{0}}$ involving the second fundamental form on $\partial B_{R_{0}}$. We claim that the sum of the last two terms vanishes. Indeed, let $D_{0}$ and $D_{0}^{*}$ be the operators obtained by replacing $M_{i}^{N}$ by $B_{R_{0}}$ in the definition of $D$ and $D^{*}$. Then by the corresponding result (4.32) for $D_{0}$, Index $D_{0}=0$, since $\mathbb{R}^{N}$ has no harmonic $L^{2}$-forms. But now the Chern-Gauss-Bonnet form vanishes, leaving us with the last two terms which are the same terms as for $D$. Hence the left-hand side of (4.32) is indeed equal to the integrated Chern-Gauss-Bonnet form. In view of the already established relation (4.14), this proves Eq. (4.15) concluding the proof of Theorem 4.6.

It remains to give a

Proof of Proposition 4.7. Let $\varphi \in \Omega_{+}\left(M_{i}^{N}, \widetilde{P}\right)$ satisfy $D \varphi=0$. For the corresponding $\psi_{1}-\psi_{2}$ we have an expansion near $\partial M_{i}^{N}$ of the form

$$
\left(\psi_{1}-\psi_{2}\right)(r)=\sum_{\mu>0} f_{\mu}(r) \tilde{\varphi}_{\mu}
$$

where $\tilde{\varphi}_{\mu}$ are normalized eigenforms of $\tilde{A}$. Since the $f_{\mu}(r)$ satisfy the equation

$$
\left(\frac{\partial}{\partial r}+\frac{\mu}{r}\right) f_{\mu}(r)=0,
$$

they are of the form

$$
f_{\mu}(r)=C_{\mu} r^{-\mu}
$$

Hence for $\varphi$ to be extendable to an even harmonic $L^{2}$-form on $M^{N}$ it suffices that the positive eigenvalues of $\tilde{A}$ all be $>\frac{1}{2}$. Conversely, an even harmonic $L^{2}$-form $\varphi$ on $M^{N}$ has an expansion of the form $\left(4.33_{+}\right)$on $\partial B_{R_{0}} \times\left[R_{0}, \infty\right)$ because terms involving $f_{\mu}(r)$ with $\mu \leqq 0$ cannot be square integrable. Hence its restriction to $M_{i}^{N}$ lies in $\operatorname{Ker} D$. Similarly let $\varphi$ be an element of $\operatorname{Ker} D^{*}$. Then for the corresponding $\left(\psi_{1}, \psi_{2}\right)$ we have

$$
\left(\psi_{1}+\psi_{2}\right)(r)=\sum_{\mu \leqq 0} f_{-\mu}(r) \cdot \tilde{\varphi}_{\mu}
$$


near $\partial B_{R_{0}}$. Hence for $\varphi$ to be extendable to an odd harmonic $L^{2}$-form on $M^{N}$ it suffices that all nonpositive eigenvalues of $\tilde{A}$ be $<-1 / 2$. Conversely the restriction of an odd harmonic $L^{2}$-form on $M^{N}$ to $M_{i}^{N}$ is an element of $\operatorname{Ker} D^{*}$. Proposition 4.7 for $N \geqq 3$ is now a consequence of

Lemma 4.8. For $N \geqq 3$ there are no eigenvalues of $\tilde{A}$ in the closed interval $[-1 / 2,+1 / 2]$.

Proof of Lemma 4.8. By the discussion in [14], the possible eigenvalues of $\tilde{A}$ are of the form

$$
\mu_{p j}^{ \pm}=\frac{(-1)^{p+1}}{2} \pm \sqrt{\lambda_{p j}+\left(p-\frac{N}{2}+1\right)^{2}}
$$

where the $\lambda_{p j}(j \geqq 0)$ are the different eigenvalues of the Laplace operator $\tilde{\Delta}$ on $\Omega^{p}\left(S^{N-1}\right)$ with nontrivial coclosed eigenforms. Also the multiplicities of the eigenvalues of $\widetilde{A}$ are computable in terms of the multiplicities of the $\lambda_{p j}$. Now all the eigenvalues of $\tilde{\Delta}$ on $\Omega^{p}\left(S^{N-1}\right)$ are of the form [43],

$$
\begin{gathered}
j(j+N-2) \text { for } p=0 \text { or } N-1, j \geqq 0 \\
(j+p-1)(j-p+N-1) \text { or }(j+p)(j-p+N-2) \text { for } 1 \leqq p \leqq N-1, j \geqq 1 .
\end{gathered}
$$

Inserting this into (4.36) immediately shows that $\widetilde{A}$ has no eigenvalues in $[-1 / 2,1 / 2]$ whenever $N>4$.

For $N=4,(4.36)$ and (4.37) show that $\mu= \pm 1 / 2$ are the only possible eigenvalues in $[-1 / 2,1 / 2]$. An explicit calculation excludes these cases.

For $N=3$, again by (4.36) and (4.37) the only possible eigenvalues in $[-1 / 2,+1 / 2]$ is zero. Again an explicit calculation excludes this possibility, completing the proof of Lemma 4.8 .

Returning to the proof of Proposition 4.7, it remains to discuss the case $N=2$. Let $z$ be the complex coordinate $z=x+i y$ for $(x, y) \in \mathbb{R}^{2}$.

Lemma 4.9. For $N=2, \tilde{A}$ has $\mu= \pm 1 / 2$ as the only eigenvalues in $[-1 / 2,+1 / 2]$. $A$ basis for the eigenforms with eigenvalue $\mu=-1 / 2$ are the two forms $1 \pm i d \theta$ $\left(\theta=\right.$ angle variable on $\left.S^{1}\right)$. For the eigenvalue $\mu=1 / 2$ a basis is given by the form $e^{i \theta}+d e^{i \theta}$ and its complex conjugate. On $C B_{R_{0}-\varepsilon}$ for $\mu=-1 / 2$ these eigenforms give rise to the harmonic 1 -forms $\frac{d z}{z}$ and $\frac{d \bar{z}}{z}$ while for $\mu=+1 / 2$ they lead to the even harmonic form $z^{-1}(1-1 / 2 d z \wedge d \bar{z})$ and its complex conjugate.

The proof is an easy exercise in linear algebra. As follows from our previous discussion, these harmonic forms on $C B_{R_{0}}$ are not in $L^{2}$, in fact their $L^{2}$-norm is logarithmically divergent.

Now as for the case $N \geqq 3$, the space of even and the space of odd harmonic forms on $M^{2}$ is linearly isomorphic to a subspace of $\operatorname{Ker} D$ and $\operatorname{Ker} D^{*}$ respectively. Conversely let $\varphi \in \operatorname{Ker} D$. Then near $\partial B_{R_{0}}, \varphi$ leads to an expansion of the form $\left(4.33_{+}\right)$. Since $\mu=0$ is not an eigenvalue, we may extend $\varphi$ to $C B_{R_{0}}$ to a harmonic form, which tends to zero at infinity. Hence the zero-form component $\varphi^{0}$ of $\varphi$ is a harmonic function on $M^{2}$, which tends to zero at infinity. But this is only possible if $\varphi^{0}=0$ [19]. Analogously the 2-form component $\varphi^{2}=\varphi-\varphi^{0}$ vanishes. Next let $\varphi$ 
be an element of $\operatorname{Ker} D^{*}$. We claim that $\varphi$ may be extended to a harmonic $L^{2}$-form on $M^{2}$. Indeed since $(d+\delta) \varphi=0$ in the interior of $M_{i}^{2} d \varphi=\delta \varphi=0$. But then by Stokes theorem

$$
\int_{\partial B_{R_{0}-\delta}} \varphi=\int_{\partial B_{R_{0}-\delta}} * \varphi=0
$$

for all small $\delta>0$ and this continues to hold in the limit $\delta \rightarrow 0$. But this means that in the expansion $\left(4.33_{-}\right)$the eigenvalue $\mu=-1 / 2$ does not give a contribution. Therefore $\varphi$ is extendable to a harmonic $L^{2}$ form on $M$ concluding the proof of Proposition 4.7.

As a consequence of Theorem 4.6 and the preceding discussion we have the following additional result for $N=2$. Let $g$ be the genus of the $k$-point compactification $\dot{M}^{2}$ of $M^{2}$ such that $\chi\left(\dot{M}^{2}\right)=2-2 g$.

Corollary 4.10. For $N=2$

$$
\operatorname{dim} H_{(2)}^{0}=\operatorname{dim} H_{(2)}^{2}=0, \quad \operatorname{dim} H_{(2)}^{1}=2 g+2 k-2 .
$$

Remark 4.11. (i) We remark that our method is not restricted to the Gauss-Bonnet operator. For example it applies equally well to the Dirac operator coupled to a vector potential. (ii) Relation (4.15) states that only the second term on the righthand side of (2.34) is nonvanishing, that is to say we have $n^{C}\left(H, H_{0}\right)=n^{\text {point }}\left(H_{0}\right)=0$ for the case at hand. Our proof of this result was based on the work of Atiyah, Patodi, and Singer, but it can also be obtained by investigating the threshold behaviour of the phase shift. In view of the correspondence of the $L^{2}$-index problem and the nonlocal boundary value problem (which is also true for the Dirac operator coupled to a vector potential), we may recover in this way the results of Atiyah, Patodi, and Singer.

\section{The Chern-Gauss-Bonnet Formula for Asymptotically Euclidean Manifolds}

In this section we shall extend the Chern-Gauss-Bonnet formula, which we obtained for manifolds euclidean at infinity as a consequence of Theorem 4.6, to asymptotically euclidean manifolds. We define them as follows.

Definition 5.1. A Riemannian manifold $\left(M^{N}, g\right)$ is called asymptotically euclidean if there is a compact submanifold $M_{i}^{N}$ such that $M^{N} \backslash M_{i}^{N}$ is a finite union of disjoint, connected open sets $O_{j}(j=1, \ldots, k)$ each of which is diffeomorphic under a map $\varphi_{j}$ to some $C B_{R_{j}}$. On each $C B_{R_{j}}$ the pull forward $g_{j}=\left(\varphi_{j}\right)_{*} g$ of the metric satisfies

$$
\begin{gathered}
C^{-1} \mathbb{1} \leqq g_{j}(x) \leqq C \mathbb{1}, \\
\left|\left(\frac{\partial}{\partial x}\right)^{\alpha}\left(g_{j, \mu \nu}(x)-\delta_{\mu \nu}\right)\right| \leqq c(1+|x|)^{-\delta-|\alpha|}
\end{gathered}
$$

for all $x \in C B_{R_{j}}$, some $C>0$, some $0<\delta \leqq 1$ and all $0 \leqq|\alpha| \leqq 2$.

In condition ( $5.1 \mathrm{~b})$ the requirement $0 \leqq|\alpha| \leqq 2$ may be replaced by the seemingly weaker condition $1 \leqq|\alpha| \leqq 2$. In fact, by condition (5.1b) for $|\alpha|=1$ and by (5.1a) there exists a positive definite matrix $g_{j}(\infty)$ such that $\left|g_{j, \mu \nu}(x)-g_{j, \mu \nu}(\infty)\right|$ $\leqq$ const $(1+|x|)^{-\delta}$. Now replace $\varphi_{j}$ by $A_{j} \circ \varphi_{j}$, where $A_{j}$ is a linear isomorphism of 
$\mathbb{R}^{N}$ such that $A_{j}^{t} g_{j}(\infty) A_{j}=\mathbb{1}$. Modifying $M_{i}^{M}, O_{j}$, and $R_{j}$ if necessary, the conditions of Definition 5.1 will be satisfied.

Such manifolds are obviously complete. Let $H_{(2)}=\bigoplus_{p=0}^{N} H_{(2)}^{p}$ be the linear space of harmonic $L^{2}$-forms on $\left(M^{N}, g\right)$ and let $\mathscr{R}(x)$ denote the Chern-Gauss-Bonnet form on $\left(M^{N}, g\right)$. Due to the assumptions (5.1) this form is integrable on $\left(M^{N}, g\right)$. The main result of this section is the following theorem, which for $N \geqq 4$ was first obtained by Stern [48] under somewhat stronger assumptions (for another alternative proof, see also [15]).

Theorem 5.2. Let $\left(M^{N}, g\right)$ be a Riemannian manifold without boundary which is asymptotically euclidean. Then all $H_{(2)}^{p}$ are finite dimensional and

$$
\sum_{p=0}^{N}(-1)^{p} \operatorname{dim} H_{(2)}^{p}=\int_{M^{N}} \mathscr{R}(x) .
$$

Proof. Let $0 \leqq \chi(x) \leqq 1$ be a $C^{\infty}$ function on $\mathbb{R}^{N}$ with support in $|x| \leqq 2$ such that $\chi(x)=1$ on $|x| \leqq 1$. For $R \geqq \max _{j} R_{j}$ let $M_{>R}^{N}=\bigcup_{j} \varphi_{j}^{-1}\left(C B_{R}\right)$. Now define $g_{R}$ to be the smooth metric on $M^{N}$ given as $g$ on the complement of $M_{>R}^{N}$ and by the pull-back via $\varphi_{j}$ to $O_{j}$ of

$$
\hat{g}_{j, \mu \nu}(x)=\delta_{\mu \nu}+\chi\left(\frac{x}{R}\right)\left\{g_{j, \mu v}(x)-\delta_{\mu v}\right\}
$$

for $x \in \varphi_{j}\left(O_{j}\right)=C B_{R_{j}}$.

Then the Riemannian manifold $\left(M^{N}, g_{R}\right)$ is euclidean at infinity. Let $\mathscr{R}_{R}(x)$ denote the resulting Chern-Gauss-Bonnet form. Obviously we have

$$
\left|\int_{M^{N}}\left(\mathscr{R}_{R}(x)-\mathscr{R}(x)\right)\right| \leqq \text { const } R^{-\delta}
$$

Let $H_{(2), R}=\bigoplus_{p=0}^{N} H_{(2), R}^{p}$ be the space of harmonic $L^{2}$-forms on $\left(M^{N}, g_{R}\right)$. Then by Theorem (4.6) we have

$$
\sum_{p=0}^{N}(-1)^{p} \operatorname{dim} H_{(2), R}^{p}=\int_{M^{N}} \mathscr{R}_{R}(x)
$$

for all $\max _{j} R_{j} \leqq R<\infty$. Hence Theorem 5.2 will be proved, once we have shown that the left-hand side of (5.5) is independent of $R \geqq \max R_{j}$ and equals the left-hand side of (5.2). To prove this, we shall employ $L^{2}$-de Rham cohomology. To abbreviate notation, let $g_{\infty}=g$ and denote by $\mathscr{H}_{R}\left(\max _{j} R_{j} \leqq R \leqq \infty\right)$ the Hilbert space of square integrable forms on $\left(M^{N}, g_{R}\right)$ with scalar product $(,)_{R}$. Let $d_{R}$ and $\delta_{R}$ denote the closures in $\mathscr{H}_{R}$ of the exterior derivative and coderivative on $\Omega_{c}\left(M^{N}\right)$. Let

$$
\begin{aligned}
E_{R} & =\overline{\operatorname{Range}}_{R}=\text { closure in } \mathscr{H}_{R} \text { of } d \Omega_{c}\left(M^{N}\right), \\
E_{R}^{*} & ={\overline{\operatorname{Range} \delta_{R}}}=\text { closure in } \mathscr{H}_{R} \text { of } \delta_{R} \Omega_{c}\left(M^{N}\right) \\
& =\text { closure in } \mathscr{H}_{R} \text { of } *_{R} d \Omega_{c}\left(M^{N}\right) .
\end{aligned}
$$


Here $*_{R}$ is the Hodge star operator on $\left(M^{N}, g_{R}\right)$. Since $\left(M^{N}, g_{R}\right)$ is complete, the space of $L^{2}$-harmonic forms $H_{(2), R}$ coincides with the space of all forms $\varphi$ in $\mathscr{H}_{R}$ satisfying $d \varphi=0$ and $\delta_{R} \varphi=0$ in the sense of distributions. Hence by a well known theorem of Kodaira [38], one has the following orthogonal direct sum decomposition

$$
\mathscr{H}_{R}=E_{R} \oplus E_{R}^{*} \oplus H_{(2), R} .
$$

Now let $\mathscr{H}_{R}^{p}$ be the closed subspace of $p$-forms in $\mathscr{H}_{R}$. Define $\bar{H}_{(2), R}=\bigoplus_{p=0}^{N} \bar{H}_{(2), R}^{p}$ with

$$
\bar{H}_{(2), R}^{p}=\operatorname{ker} d_{R} \cap \mathscr{H}_{R}^{p} / E_{R} \cap \mathscr{H}_{R}^{p}
$$

to be the reduced $L^{2}$-de Rham cohomology group. Employing (5.6) we obtain

$$
\bar{H}_{(2), R}^{p} \cong H_{(2), R}^{p}
$$

for all $0 \leqq p \leqq N, \max _{j} R_{j} \leqq R \leqq \infty$.

Now the crucial observation is that the norms on $\Omega_{c}\left(M^{N}\right)$ given by the scalar products $(,)_{R}$ are all equivalent. In particular the Banach spaces $\mathscr{D}\left(d_{R}\right)$ with the norm

$$
\|\varphi\|_{d_{R}}=\left((\varphi, \varphi)_{R}+\left(d_{R} \varphi, d_{R} \varphi\right)_{R}\right)^{1 / 2}
$$

are naturally isomorphic as topological vector spaces. Hence the spaces $\bar{H}_{(2), R}^{p}$ are all isomorphic and coincide with $H_{(2)}^{p}=H_{(2), \infty}^{p}$. This concludes the proof of Theorem 5.2.

Remark 5.3. Note that we actually proved a stronger result than given by Theorem 5.2. In fact the conclusion of Theorem 5.2 holds for any metric where the condition (5.1b) is replaced by the requirements that (i) $\mathscr{R}(x)$ is integrable and (ii) the left-hand side of (5.4) tends to zero as $R \rightarrow \infty$.

So far we have not yet discussed the scattering theory with spaces which are asymptotically euclidean. However, based on the known results for scattering in the space of 0 -forms (like absence of a singular continuous spectrum, absence of discrete eigenvalues above threshold and unitarity of the Møller operators, see [21]), we expect all results valid in the context of spaces which are euclidean at infinity to carry over to this more general situation.

Acknowledgements. We have profited from discussions with J. Brüning, V. Enss, and B. Schroer. The visit of one of us (N.B.) at the Freie Universität Berlin, Berlin (West), was made possible through a joint exchange program with Leningrad State University. He would like to thank the Physics Department for its hospitality.

\section{References}

1. Alvarez-Gaumé, L.: Supersymmetry and the Atiyah-Singer index theorem. Commun. Math. Phys. 90, 161-173 (1983)

2. Alves, N.A., Aratyn, H., Zimerman, A.H.: $\beta$ dependence of the Witten index. Phys. Lett. B 173, 327-331 (1986) 
3. Andrianov, A.A., Borisov, N.V., Ioffe, M.V.: Nonstationary approach to scattering theory for supersymmetric Hamiltonians in quantum mechanics and supersymmetry of nuclear interactions. Preprint FUB-HEP/86-6, Phys. Lett. B 181, 141-144 (1986) and Theor. Mat. Fiz. (to appear) (in Russian)

4. Aronszajn, N.: A unique analytic continuation theorem for elliptic partial differential equations or inequaltiies of second order. J. Math. Pure Appl. 36, 235-250 (1957)

5. Atiyah, M.F.: Elliptic operators, discrete groups and von Neumann algebras. Astérisque. Soc. Math. France 32-33, 43-72 (1961)

6. Atiyah, M.F., Bott, R., Patodi, V.K.: On the heat equation and the index theorem. Invent. Math. 19, 279-330 (1973)

7. Atiyah, M.F., Patodi, V.K., Singer, I.M.: Spectral asymmetry and Riemannian geometry. I. Math. Proc. Camb. Phil. Soc. 77, 43-69 (1975)

8. Barbasch, D., Moscovici, H.: $L^{2}$-index and the Selberg trace formula. J. Funct. Anal. 53, 151-201 (1983)

9. Baumgärtel, H., Wollenberger, M.: Mathematical scattering theory. Basel, Boston, Stuttgart: Birkhäuser 1983

10. Beth, E., Uhlenbeck, G.E.: Quantum theory of the non-ideal gas. Part II. Behaviour at low temperatures. Physica 4, 915-924 (1937)

11. Bollé, D., Gesztesy, F., Grosse, H., Simon, B.: Krein's spectral shift function and Fredholm determinants as efficient methods to study supersymmetric quantum mechanics. Preprint, June, 1986

12. Bott, R., Seeley, R.: Some remarks on the paper of Callias. Commun. Math. Phys. 62, 235-245 (1978)

13. Boyanovsky, D., Blankenbecler, R.: Fractional indices in supersymmetric theories. Phys. Rev. D 30, 1821-1824 (1984)

14. Brüning, J., Seeley, R.: An index theorem for first order regular operators. Preprint Univ. Augsburg 1986

15. Brüning, J., Seeley, R.: Index theory for regular singular operators and applications (to appear)

16. Buslaev, V.S.: Scattered plane waves, spectral asymptotics and trace formulas in exterior domains. Dokl. Akad. Nauk SSSR 197, 999-1002 (1971)

17. Buslaev, V.S.: On the asymptotic behavior of the spectral characteristics of exterior problems for the Schrödinger operator. Izv. Akad. Nauk SSSR Ser. Mat. 39, No. 1 (1975) [Math. USSR Izv. 9, 139-223 (1975)]

18. Callias, C.: Axial anomalies and index theorems on open spaces. Commun. Math. Phys. 62, 213-234 (1978)

19. Chavel, I.: Eigenvalues in Riemannian geometry. London: Academic Press 1984

20. Conner, P.E.: The Neumann's problem for differential forms on Riemannian manifolds. Mem. Am. Math. Soc. 20 (1956)

21. Cotta-Ramusino, P., Krüger, W., Schrader, R.: Quantum scattering by external metrics and Yang-Mills potentials. Ann. Inst. H. Poincaré 31, 43-71 (1979)

22. Davies, E.B.: On Enss' approach to scattering theory. Duke Math. J. 47, 171-185 (1980)

23. D'Olivo, J.C., Urrutia, L.F., Zertuche, F.: Study of a three-dimensional quantum-mechanical model with nucleon-nucleon-type interaction. Phys. Rev. D 32, 2174-2189 (1985)

24. Enss, V.: Geometric methods in spectral and scattering theory of Schrödinger operators. Rigorous Atomic and Molecular Physics. Velo, G., Wightman, A.S. (eds.). Nato advanced study Inst. Ser., Ser. B Physics, Vol. 74, Erice, 1980. New York, London: Plenum Press 1981

25. Fedosov, B.V.: Analytic formulas for index of elliptic operators. Tr. Mosk. Mat. O 30, 159-240 (1974) (in Russian)

26. Friedan, D., Windey, P.: Supersymmetric derivation of the Atiyah-Singer index and the chiral anomaly. Nucl. Phys. B 235, 395-416 (1984)

27. Friedman, A.: Partial differential equations of parabolic type. Englewood Cliffs, New York: Prentice Hall 1964

28. Gaffney, M.P.: The heat equation method of Milgram and Rosenbloom for open Riemannian manifolds. Ann. Math. 60, 458-466 (1954) 
29. Gesztesy, F.: Scattering theory for one-dimensional systems with nontrivial spatial asymptotics. Lecture Notes in Mathematics. Berlin, Heidelberg, New York: Springer (to appear)

30. Gilkey, P.B.: Invariance theory, the heat equation, and the Atiyah-Singer index theorem. Wilmington: Publish or Perish 1984

31. Gromov, N., Lawson, H.B., Jr.: Positive scalar curvature and the Dirac Operator on complete Riemannian manifolds. Publ. Math. IHES 58, 295-408 (1983)

32. Guillopé, L.: Asymptotique de la phase de diffusion pour l'operateur de Schrödinger avec potenticl. C.R. Acad. Sci. Paris 293, Ser. I, 601-603 (1981)

33. Jensen, A., Kato, T.: Asymptotic behaviour of the scattering phase for exterior domain. Commun. Partial Differ. Equations 3, 1165-1195 (1978)

34. Kac, M.: Can one hear the shape of a drum?. Am. Math. Mon. 73, 1-23 (1966)

35. Kato, T.: Wave operators and unitary equivalence. Pac. J. Math. 15, 171-180 (1965)

36. Kato, T.: Scattering theory with two Hilbert spaces. J. Funct. Anal. 1, 342-369 (1967)

37. Kato, T.: Perturbation theory for linear operators. Berlin, Heidelberg, New York: Springer 1980

38. Kodaira, K.: Harmonic fields in Riemannian manifolds. Ann. Math. 50, 587-665 (1949)

39. Majda, A., Taylor, M.: Inverse scattering problems for transparent obstacles, electromagnetic waves, and hyperbolic systems. Commun. Partial Differ. Equations 2 (4), 395-438 (1977)

40. Majda, A., Ralston, R.: An analogue of Weyl's theory for unbounded domains. I. Duke Math. J. 45, 183-196 (1978); II. ibid 45, 513-536 (1978); III. ibid 45, 725-731 (1979)

41. Müller, W.: Manifolds with cusps of rank one. Lecture Notes in Mathematics, Vol. 1244. Berlin, Heidelberg, New York: Springer 1987

42. Niemi, A.J., Semenoff, G.W.: Index theorems on open infinite manifolds. Nucl. Phys. B 269, 131-159 (1986)

43. Paquet, L.: Méthode de separation des variables et calcul du spectre d'operateurs sur les formes differentielles. Bull. Sci. Math., $2^{\mathrm{e}}$ ser. 105, 85-112 (1981)

44. Perry, P.A.: Mellin transforms and scattering theory. I. Short range potentials. Duke Math. J. 47, 187-193 (1980)

45. Ray, D.B., Singer, I.M.: R-Torsion and the Laplacian on Riemannian manifolds. Adv. Math. 7, 145-210 (1971)

46. Schrader, R.: High energy behaviour for nonrelativistic scattering by stationary external metrics and Yang Mills potentials. Z. Phys. C 4, 27-36 (1980)

47. Spanier, E.H.: Algebraic topology. New York: McGraw-Hill 1966

48. Stern, M.A.: $L^{2}$-index theorems on warped products. Dissertation, Princeton Univ. 1984

49. Taubes, C.H.: Stability in Yang-Mills theories. Commun. Math. Phys. 91, 235-263 (1983)

50. Vilenkin, N.Ja.: Functions speciales et theorie de la representation des groups. Paris: Dunod 1969

51. Werner, P.: Spectral properties of the Laplace operator with respect to electric and magnetic boundary conditions. J. Math. Anal. Appl. 92, 1-65 (1983)

52. Witten, E.: Constraints on supersymmetry breaking. Nucl. Phys. B 202, 253-316 (1982)

53. Witten, E.: Supersymmetry and Morse theory. J. Differ. Geom. 17, 661-692 (1982)

54. Donnelly, H.: Essential spectrum and heat kernel estimates (preprint)

Communicated by C. H. Taubes

Received October 22, 1986; in revised form July 15, 1987 
